\title{
Higher form symmetries and M-theory
}

\author{
Federica Albertini, ${ }^{a}$ Michele Del Zotto, ${ }^{a, b}$ Iñaki García Etxebarria ${ }^{a}$ \\ and Saghar S. Hosseini ${ }^{a}$ \\ ${ }^{a}$ Department of Mathematical Sciences, Durham University, \\ Durham, DH1 3LE, United Kingdom \\ ${ }^{b}$ Department of Mathematics, and Department of Physics and Astronomy, Uppsala University, \\ Uppsala, Sweden \\ E-mail: federica.albertini@durham.ac.uk, michele.delzotto@math.uu.se, \\ inaki.garcia-etxebarria@durham.ac.uk, \\ sagharsadat.hosseinisemnani@durham.ac.uk
}

ABSTRACT: We discuss the geometric origin of discrete higher form symmetries of quantum field theories in terms of defect groups from geometric engineering in M-theory. The flux non-commutativity in M-theory gives rise to (mixed) 't Hooft anomalies for the defect group which constrains the corresponding global structures of the associated quantum fields. We analyze the example of $4 \mathrm{~d} \mathcal{N}=1 \mathrm{SYM}$ gauge theory in four dimensions, and we reproduce the well-known classification of global structures from reading between its lines. We extend this analysis to the case of $7 \mathrm{~d} \mathcal{N}=1 \mathrm{SYM}$ theory, where we recover it from a mixed 't Hooft anomaly among the electric 1-form center symmetry and the magnetic 4-form center symmetry in the defect group. The case of five-dimensional SCFTs from M-theory on toric singularities is discussed in detail. In that context we determine the corresponding 1-form and 2-form defect groups and we explain how to determine the corresponding mixed 't Hooft anomalies from flux non-commutativity. Several predictions for non-conventional 5d SCFTs are obtained. The matching of discrete higher-form symmetries and their anomalies provides an interesting consistency check for $5 \mathrm{~d}$ dualities.

Keywords: Field Theories in Higher Dimensions, Global Symmetries, M-Theory, Supersymmetric Gauge Theory

ArXiv EPrint: 2005.12831 


\section{Contents}

1 Introduction 1

1.1 Discrete higher form symmetries from M-theory: general philosophy 2

1.2 Summary of results and structure of this work 4

2 Global structures of $\mathcal{N}=1$ seven-dimensional theories from M-theory 6

3 Higher form symmetries and non-commutative fluxes for 5d SCFTs 9

$\begin{array}{lll}3.1 & \text { Field theory analysis } & 10\end{array}$

$\begin{array}{lll}3.2 & \text { M-theory and higher form symmetries } & 11\end{array}$

$\begin{array}{lll}3.3 & \text { The case of toric Calabi-Yau varieties } & 14\end{array}$

$\begin{array}{llr}4 \mathfrak{s u}(p)_{k} \text { theory } & 15\end{array}$

$\begin{array}{lll}4.1 & \text { Toric realization and geometric computation } & 16\end{array}$

4.2 Non-vanishing Chern-Simons levels and the charge of instanton particles 17

$\begin{array}{ll}4.3 \text { Adding fundamental matter } & 19\end{array}$

5 Higher form symmetries and 5d duality 20

$\begin{array}{lll}5.1 \text { The beetles } & 20\end{array}$

5.2 Dualities and modified prescription for non-isolated toric singularities 23

6 Non-Lagrangian examples $\quad \mathbf{2 5}$

6.1 The global structure of the 5d $E_{0}$ SCFT 26

6.2 The higher rank $5 \mathrm{~d} E_{0}$ theories 28

$\begin{array}{ll}6.3 \text { The } 5 \mathrm{~d} \text { trinions and their descendants } & 29\end{array}$

6.4 More about 5d "dualities" 30

7 Global structure of $4 \mathrm{~d} \mathcal{N}=1$ theories and M-theory on $G_{2}$ spaces 32

7.1 Example: $4 \mathrm{~d} \mathcal{N}=1 \mathrm{SYM}$

A The Mori cone for $\mathcal{C}_{\mathbb{R}}\left(Y^{p, q}\right) \quad 35$

$\begin{array}{ll}\text { B Linking forms } & 36\end{array}$

$\begin{array}{lll}\text { B.1 } & \mathcal{C}_{\mathbb{R}}\left(Y^{p, q}\right) & 36\end{array}$

B.2 $\mathbb{C}^{3} / \mathbb{Z}_{2 n+1}$ 


\section{Introduction}

In this paper we are interested in the study of discrete higher form symmetries for quantum field theories that arise by geometric engineering in M-theory. In full generality, $p$ branes wrapping non-compact $k$-cycles in the non-compact internal geometry $\mathcal{V}$ of geometric engineering give rise to $p-k+1$ dimensional defects for the field theory. Similarly, $p$ branes wrapping compact $k$-cycles give rise to $p-k+1$ BPS degrees of freedom. Generalizing the 't Hooft screening argument along the lines of [1], for every $p$ brane wrapping a non-compact $k$-cycle we expect to find a corresponding defect group of discrete higher $(p-k+1)$-form symmetries, ${ }^{1}$

$$
\mathbb{D}:=\bigoplus_{n} \mathbb{D}^{(n)} \quad \text { where } \quad \mathbb{D}^{(n)}:=\bigoplus_{\substack{p \text { branes and } k \text { cycles } \\ \text { such that } p-k+1=n}} \operatorname{Tor}\left(\frac{H_{k}(\mathcal{V}, \partial \mathcal{V})}{H_{k}(\mathcal{V})}\right)
$$

The operators that are measuring these charges are the flux operators in the string theory we are adopting for the engineering that are sourced by the corresponding kind of $p$ brane. These operators are the charge operators for the $\mathbb{D}^{(p-k+1)}$ factor of the defect group. We stress that $\mathbb{D}$ is not the group of higher form symmetries of a quantum field theory yet, rather it is the group of higher form symmetries acting on the geometric engineering Hilbert space, which is the Hilbert space that the given string theory assigns to the given noncompact geometry.

The geometric engineering formalism indeed has a deeper insight on the structure of the resulting field theories: whenever the internal manifold has torsional cycles, we have non-commuting fluxes (a fact that has been thoroughly discussed in the seminal papers by Freed, Moore and Segal [6, 7] — see also [8]). Correspondingly, pairs of electromagnetically dual branes in the geometric engineering give rise to mixed 't Hooft anomalies among different higher form symmetry factors of the defect group.

Examples including the computation of such mixed 't Hooft anomalies in the context of the $6 \mathrm{~d} 2$-form symmetry defect group appeared in $[1,8-10] .{ }^{2}$ The purpose of the present paper is to extend this study in the context of models geometrically engineered within Mtheory. Our main aim is to explain how the discrete higher form symmetries arise in this context and what is their relation with the M-theory defect group. It is well-known that geometric engineering in string theory gives an alternative formulation of field theories that often proves useful when studying models that cannot be realized perturbatively, which is the case for all SCFTs in dimension higher than four [14, 15]. Among the most interesting examples in this class therefore are 5d SCFTs, whose geometric engineering [16-20] has seen a lot of recent developments (for an incomplete list of recent references see [21-68]). Among the applications of our formalism, we determine the global structure and the discrete higher form symmetries of 5d SCFTs. A more detailed summary of our results can be found in section 1.2 below.

\footnotetext{
${ }^{1}$ See $[2-4]$ for a definition of higher form symmetries. In this paper we adopt the Córdova-DumitrescuIntriligator notation: $G^{(m)}$ denotes an $m$-form symmetry group $G$ [5]. We stress that in principle one could consider to include the non-torsional part of the groups in the definition (1.1). We choose not to include it to avoid cluttering notation below. Moreover, there might be other global symmetries arising from isometries which the defect group will not detect — we thank Kantaro Ohomori for this remark.

${ }^{2}$ See also [11-13] for some deep implications.
} 


\subsection{Discrete higher form symmetries from M-theory: general philosophy}

In this work we consider the SQFTs obtained from M-theory via geometric engineering on backgrounds of the form

$$
\mathcal{M}_{11}=\mathcal{M}_{D} \times \mathcal{V}_{d} \quad d+D=11
$$

where $\mathcal{V}_{d}$ is a local internal geometry for M-theory and $\mathcal{M}_{D}$ is a $D$ dimensional space time manifold where the geometrically engineered $D$ dimensional quantum field theory $\mathcal{T}_{\mathcal{V}_{d}}$ lives. For simplicity, we assume: ${ }^{3}$

- $\mathcal{V}_{d}$ is a supersymmetric background, therefore $\mathcal{T}_{\mathcal{V}_{d}} \in \mathrm{SQFT}_{D}$; moreover

$$
\mathcal{V}_{d}=\mathcal{C}\left(Y_{d-1}\right)
$$

meaning that $\mathcal{V}_{d}$ is a metric cone over a $d-1$ dimensional manifold $Y_{d-1}$;

- $\mathcal{M}_{D}$ is a closed spin manifold without torsion.

Naively, this M-theory setup computes the partition function of the theory $\mathcal{T}_{\mathcal{V}_{d}}$ on the manifold $\mathcal{M}_{D}$,

$$
Z_{\mathcal{T}_{\mathcal{V}_{d}}}\left(\mathcal{M}_{D}\right) \in \mathbb{C}
$$

The latter should be fully specified by the M-theory background, but in presence of mixed 't Hooft anomalies for the factors in the defect group not all fluxes can be diagonalized simultaneously, thus leading to several different choices. These choices are in one-to-one correspondence with the possible global structures of the quantum field theory $\mathcal{T}_{\mathcal{V}_{d}}$.

The main feature of the geometric engineering limit is that the internal manifold is non-compact, and therefore the M-theory background has a boundary at infinity

$$
\partial \mathcal{M}_{11}=\mathcal{M}_{D} \times \partial \mathcal{V}_{d}
$$

If this is the case, we can consider a Hamiltonian quantization viewing the direction normal to the boundary as time, and assign to this system a Hilbert space

$$
\mathcal{H}_{M}\left(\partial \mathcal{M}_{11}\right)
$$

By analogy with the IIA and IIB superstrings, the Hilbert space $\mathcal{H}_{M}\left(\partial \mathcal{M}_{11}\right)$ should have a grading in terms of the $M$-theory generalized cohomology theory group, which we denote $\mathbb{E}_{M}\left(\partial \mathcal{M}_{11}\right)$. The group $\mathbb{E}_{M}\left(\partial \mathcal{M}_{11}\right)$ is expected to parametrize the flux sectors of M-theory. Classically one would expect all such fluxes to commute, but this is not the case because the group $\mathbb{E}_{M}\left(\partial \mathcal{M}_{11}\right)$ can contain a torsional part

$$
\text { Tor } \mathbb{E}_{M}\left(\partial \mathcal{M}_{11}\right)=\left\{x \in \mathbb{E}_{M}\left(\partial \mathcal{M}_{11}\right): n x=0 \text { for some } n \in \mathbb{Z}\right\}
$$

\footnotetext{
${ }^{3}$ Both assumptions can be dropped in principle, but we want to work in the simplest possible setup that highlights the features we want to study.
} 
Under the assumptions above, there is a simple connection in between the defect group and Tor $\mathbb{E}_{M}\left(\partial \mathcal{M}_{11}\right)$ :

$$
\text { Tor } \mathbb{E}_{M}\left(\partial \mathcal{M}_{11}\right)=\bigoplus_{j} H^{j+1}\left(\mathcal{M}_{D}\right) \otimes \mathbb{D}^{(j)}
$$

In presence of torsional fluxes we expect to have a grading of the geometric engineering Hilbert space in terms of

$$
\mathcal{H}_{M}=\bigoplus_{\boldsymbol{\alpha} \in \mathbb{E}_{M}^{o}\left(\partial \mathcal{M}_{11}\right)} \mathcal{H}_{M}(\boldsymbol{\alpha}) \quad \text { with } \quad \mathbb{E}_{M}^{o}\left(\partial \mathcal{M}_{11}\right) \equiv \frac{\mathbb{E}_{M}\left(\partial \mathcal{M}_{11}\right)}{\text { Tor } \mathbb{E}_{M}\left(\partial \mathcal{M}_{11}\right)}
$$

where each factor $\mathcal{H}_{M}(\boldsymbol{\alpha})$ is in turn a representation of a Heisenberg algebra of torsional fluxes of the form

$$
\Psi_{x} \Psi_{y}=s(x, y) \Psi_{y} \Psi_{x}
$$

where

$$
s: \text { Tor } \mathbb{E}_{M}\left(\partial \mathcal{M}_{11}\right) \times \text { Tor } \mathbb{E}_{M}\left(\partial \mathcal{M}_{11}\right) \rightarrow \mathrm{U}(1)
$$

is a perfect pairing. This pairing encodes the mixed 't Hooft anomalies among the higher form symmetries of the geometric engineering Hilbert space. Abusing language, in light of (1.8) we will refer to these as the mixed 't Hooft anomalies for the defect group. Since the flux operators do not commute we cannot specify the asymptotic values for all fluxes simultaneously: two steps are required

1. We need to choose a maximally isotropic subgroup $L \subset$ Tor $\mathbb{E}_{M}\left(\partial \mathcal{M}_{11}\right)$ of fluxes that can be simultaneously measured;

2. We need to choose a "zero flux" state, which corresponds to the unit eigenvalue

$$
\Psi_{x}|0, L\rangle=|0, L\rangle \quad \forall x \in L
$$

Then we obtain a basis for the geometric engineering Hilbert space parametrized by

$$
|f, L\rangle:=\Psi_{f}|0, L\rangle \quad f \in \mathrm{F}_{L}:=\frac{\text { Tor } \mathbb{E}_{M}\left(\partial \mathcal{M}_{11}\right)}{L} .
$$

A choice of background fluxes for the higher form symmetries of this theory corresponds to fixing a state

$$
\left|\left\{a_{f}\right\}\right\rangle=\sum_{f \in \mathrm{F}_{L}} a_{f}|f, L\rangle
$$

whence the corresponding partition function is determined: the open manifold $\mathcal{M}_{11}$ can be viewed as an element $\left\langle\mathcal{M}_{11}\right|$ of $\mathcal{H}_{M}\left(\partial \mathcal{M}_{11}\right)^{*}:=\operatorname{Hom}\left(\mathcal{H}_{M}\left(\partial \mathcal{M}_{11}\right), \mathbb{C}\right)$ so the partition function is $\left\langle\mathcal{M}_{11} \mid\left\{a_{f}\right\}\right\rangle$ - see [8] for a more detailed version of this argument.

We stress that to fully specify a quantum field theory $\mathcal{T}_{\mathcal{V}_{d}}$ these two steps are required. Indeed only in this case we end up with a partition function. Without specifying these details, the geometric engineering Hilbert space knows only about the whole set of possible theories that have the same local dynamics but different global structures. When we choose 
the theory corresponding to the state $|0, L\rangle$, the defects with charges in $L$ are non-genuine, while the ones with charges in $F_{L}$ are the genuine ones (see section 3.3 of [8]). When we specify the state $|0, L\rangle$ this breaks the defect group $\mathbb{D}$ to the higher form symmetry group of the genuine defects of the corresponding quantum field theory $\mathcal{T}_{\mathcal{V}_{d}}$. The operators $\Psi_{f}$ generate background flux for the higher form symmetry associated to the genuine operators. We can think of them as domain walls and of the states $|f, L\rangle$ as labeling distinct superselection sectors.

To clarify this statement let's consider the example of geometric engineering of $4 \mathrm{~d}$ $\mathcal{N}=1 \mathrm{SYM}$ with simple simply-laced Lie algebra $\mathfrak{g}_{\Gamma}$ where $\Gamma \subset \mathrm{SU}(2)$. The defect group we obtain for this geometry is

$$
\mathbb{D}=Z\left(G_{\Gamma}\right)_{M 2}^{(1)} \oplus Z\left(G_{\Gamma}\right)_{M 5}^{(1)}
$$

We have an electric and a magnetic one form symmetry valued in the center of $G_{\Gamma}$, the universal cover group. These one-form symmetries of the geometric engineering Hilbert space however have a mixed 't Hooft anomaly: the corresponding charge operators do not commute. More precisely, for fluxes labelled by $a_{i}=(\omega \otimes \ell)_{i} \in H^{2}\left(\mathcal{M}_{4}\right) \otimes Z\left(G_{\Gamma}\right)$ we have

$$
\Phi_{M 2, a_{1}} \Psi_{M 5, a_{2}}=\exp \left(2 \pi i \mathrm{~L}_{\Gamma}\left(\ell_{1}, \ell_{2}\right) \int_{\mathcal{M}_{4}} \omega_{1} \wedge \omega_{2}\right) \Psi_{M 5, a_{2}} \Phi_{M 2, a_{1}}
$$

where $\mathrm{L}_{\Gamma}\left(\ell_{1}, \ell_{2}\right)$ is a perfect pairing in $Z\left(G_{\Gamma}\right)$ that is determined below - see table 1 . Specifying a state now selects a surviving subgroup of $\mathbb{D}$ which become the 1-form symmetry for the SYM quantum field theory. Consider for example the case $\Gamma=\mathbb{Z}_{N}$. For instance, we can choose a maximal isotropic lattice $L_{M 2}$ generated by the M2 flux operators. Then the state $\left|0, L_{M 2}\right\rangle$ corresponds to the theory $\operatorname{PSU}(N)=\mathrm{SU}(N) / \mathbb{Z}_{N}$ and the wrapped $M 5$ branes are the genuine defects charged under the resulting magnetic 1-form symmetry. Conversely choosing to set to zero all M5 fluxes we are selecting the state $\left|0, L_{M 5}\right\rangle$ : we are preserving the electric 1-form symmetry, thus leading to the theory with gauge group $\mathrm{SU}(N)$.

\subsection{Summary of results and structure of this work}

The structure of this work is as follows. As a simpler warm-up example, in section 2 we discuss the case of $7 \mathrm{~d}$ gauge theories with simple simply-laced lie groups $G \in A D E$; of course, we find agreement with the global structure obtained by considering Wilson and 't Hooft operators in the $7 \mathrm{~d}$ side, and the global structure predicted by M-theory flux non-commutativity. In section 3 we set the stage for our analysis of the global structure of 5d SCFTs from M-theory on canonical CY singularities. In this work, for simplicity, we consider geometries with some constraints on the cohomology which restrict the structure of the models we analyze. As a result we obtain 5 d defect groups of the form ${ }^{4}$

$$
\mathbb{D}=\left(\mathbf{Z}_{M 2}^{(1)} \oplus \mathbf{Z}_{M 5}^{(2)}\right) \oplus\left(\mathbf{Z}_{M 2}^{(-1)} \oplus \mathbf{Z}_{M 5}^{(4)}\right)
$$

\footnotetext{
${ }^{4}$ In principle one could expect to obtain a further factor $\tilde{Z}_{M 2}^{(2)} \oplus \tilde{\mathbf{Z}}_{M 5}^{(1)}$, for geometries with nontrivial $H_{1}\left(\mathcal{V}_{6}\right)$ and $H_{5}\left(\mathcal{V}_{6}\right)$ that we assume to vanish for simplicity in chasing exact sequences.
} 
that often present very interesting global structures arising from the mechanism we have outlined above. Here $\mathbf{Z}$ is an abelian discrete group given by the torsional part of the cokernel of the intersection matrix of the corresponding CY. Physically this intersection matrix is associated to the Dirac pairing among the monopole strings and the BPS particles of the SCFT in a Coulomb phase and Z measures the 't Hooft charges of the defects. We begin reviewing some field theory results, and then proceed determining the corresponding defect groups from geometry first in general, and then focussing on the case of toric canonical CY singularities, that are the main class of examples we use for this project. In section 4 the case of the 5d Yang-Mills theories with gauge algebra $\mathfrak{s u}(p)$ and Chern-Simons level $k$ is studied in detail, as a consistency check for our methods. In section 5 we discuss applications of the higher form symmetries to the study of $5 \mathrm{~d}$ dualities among different gauge theory phases of 5d SCFTs. Exploiting consistency with dualities we extend (and prove) a new purely graphical prescription to compute the defect groups for arbitrary toric CY singularities. We proceed determining the defect groups for several examples of $5 \mathrm{~d}$ SCFTs with non-trivial flavor symmetries, corresponding to non-isolated singularities, that we study in section 6 . An interesting class of examples that we consider is given by the higher rank generalizations of the $E_{0} 5 \mathrm{~d}$ SCFT. We find that the corresponding geometry gives a defect group

$$
\mathbb{D}=\left(\mathbb{Z}_{2 r+1}\right)_{M 2}^{(1)} \oplus\left(\mathbb{Z}_{2 r+1}\right)_{M 5}^{(2)}
$$

with a nontrivial global structure with pairing $1 /(2 r+1)$. From this result it follows that there are as many inequivalent versions of the $5 \mathrm{~d} E_{0}^{(r)}$ theories as there are inequivalent versions of $\mathfrak{s u}(2 r+1)$ in $4 \mathrm{~d}$. These models enjoy different combinations of the electric/magnetic 1-form and 2-form symmetries in the defect group. In particular we have a purely electric $5 \mathrm{~d} E_{0}^{(r)}$ theory which has higher form symmetry $\mathbb{Z}_{2 r+1}^{(1)}$ and a purely magnetic $5 \mathrm{~d} E_{0}^{(r)}$ theory that has higher form symmetry $\mathbb{Z}_{2 r+1}^{(2)}$. These models have identical spectra of local operators, but different properties which can be detected from the spectrum of nonlocal operators. Using our results we show that the $5 \mathrm{~d}$ trinions (the $T_{N}$ theories) have trivial defect groups in agreement with a result by Tachikawa [69]. However, inspecting theories that can be obtained by massive deformations of the $T_{N}$ theories it is easy to find models that have much larger defect groups, for instance we find a family of models that have

$$
\mathbb{D}=\left(\mathbb{Z}_{N^{2}-3(N-1)}\right)_{M 2}^{(1)} \oplus\left(\mathbb{Z}_{N^{2}-3(N-1)}\right)_{M 5}^{(2)}
$$

We conclude the discussion with some applications of $5 \mathrm{~d}$ dualities and higher form symmetries to constrain the properties of the spectrum of operators of several strongly interacting 5d SCFTs that do not admit gauge theory phases. In section 7 we discuss some appetizer about the case of $4 \mathrm{~d} \mathcal{N}=1$ theories arising from $G_{2}$ spaces. We find that the defect group of $4 \mathrm{~d} \mathcal{N}=1$ models from M-theory has the structure

$$
\mathbb{D}=\left(\mathbf{Z}_{M 2}^{(1)} \oplus \mathbf{Z}_{M 5}^{(1)}\right) \oplus\left(\tilde{\mathbf{Z}}_{M 2}^{(0)} \oplus \tilde{\mathbf{Z}}_{M 5}^{(2)}\right) \oplus\left(\tilde{\mathbf{Z}}_{M 2}^{(-1)} \oplus \tilde{\mathbf{Z}}_{M 5}^{(3)}\right)
$$

Exploiting this result we correctly reproduce the global structure of $4 \mathrm{~d} \mathcal{N}=1$ pure $\mathrm{SYM}$ theories with gauge algebra $\mathfrak{g}$ from geometry - these models have $\tilde{Z}=0$ and $\mathrm{Z}=Z\left(G_{\Gamma}\right)$ above. In general, however, $4 \mathrm{~d} \mathcal{N}=1$ theories admit a richer global structure consisting of 
two commuting Heisenberg algebras $(Z \neq \tilde{Z}$ above) we also discuss some of the implications of this remark.

Note added. while this work was in preparation, we learned about [70] that overlaps with some of our results. We thank the authors of that paper for agreeing to coordinate the submission with us. Also, soon after this paper appeared in the arXiv the paper [71] also appeared, where the global structure of $6 \mathrm{~d}$ SCFTs coming from F-theory was studied.

\section{Global structures of $\mathcal{N}=1$ seven-dimensional theories from M-theory}

We start with the case of seven dimensional $\mathcal{N}=1$ theory with gauge algebra $\mathfrak{g}_{\Gamma}$, with $\Gamma \subset \mathrm{SU}(2)$ an ADE group. ${ }^{5}$ Such theories can be engineered by considering M-theory on $\mathcal{M}_{11}=\mathcal{M}_{7} \times \mathbb{C}^{2} / \Gamma$, with $\Gamma$ a discrete subgroup of $\mathrm{SU}(2){ }^{6}$

From the field theory side, we expect to have a one-form symmetry measuring Wilson lines, and a $7-3=4$-form symmetry measuring 't Hooft surfaces (which are operators wrapping 4-surfaces in seven dimensions). Or slightly more generally, we have electric charge operators of dimension $7-1-1=5$, associated to elements of $H^{2}\left(\mathcal{M}_{7}\right)$, measuring the flux that would be created by Wilson lines, and magnetic charge operators of dimension $7-4-1=2$, associated to elements of $H^{5}\left(\mathcal{M}_{7}\right)$, measuring the flux created by 't Hooft surfaces. It is useful to make this distinction, since on manifolds of non-trivial topology it is possible to introduce the fluxes without introducing the extended operators themselves. These extended charge operators are valued on $Z\left(G_{\Gamma}\right)$, the centre of the universal cover of any gauge group with algebra $\mathfrak{g}_{\Gamma}$. For the $\operatorname{ADE}$ cases we have $Z\left(G_{\Gamma}\right)=\Gamma^{\mathrm{ab}}:=[\Gamma, \Gamma]$, the abelianization of $\Gamma$ (see table 1 below).

Not all such higher form symmetries are present in any given theory simultaneously, though: since the Wilson line operators are not mutually local with respect to the 't Hooft surfaces it is not possible to construct charge operators measuring all such charges at the same time. What we can do instead is — as in $[76,77]$ — to choose a maximal set of mutually local Wilson/'t Hooft operators, and declare that these are the genuine ones.

We refer to the choice of $p$-form charge operators present in the theory as a choice of global form for the theory and an actual choice of flux for these operators as a background for the $p$-form symmetry in that theory. If we sum over fluxes in $H^{2}\left(\mathcal{M}_{7} ; \Gamma^{\mathrm{ab}}\right)_{m}$ we would have the $G_{\Gamma} / \Gamma$ theory $\left(\mathrm{SU}(N) / \mathbb{Z}_{N}\right.$, for instance, in this context the fluxes are often known as the generalized Stiefel-Whitney classes of the bundle), with a 4-form symmetry, while if we sum over $H^{5}\left(\mathcal{M}_{7} ; \Gamma^{\mathrm{ab}}\right)_{e}$ instead we have the $G_{\Gamma}$ theory ( $\mathrm{SU}(N)$, for instance) with a 1-form symmetry. We emphasize that in a purely perturbative presentation the notion of "sum over fluxes in $H^{5}\left(\mathcal{M}_{7} ; \Gamma^{\mathrm{ab}}\right)_{e}$ " is somewhat formal, as there are no fields in the Lagrangian that can detect these fluxes. Nevertheless this choice of language becomes very

\footnotetext{
${ }^{5}$ It is possible to consider non-simply-laced cases too in M-theory using frozen fluxes [72]. We assume that no such fluxes are present, but it would be certainly be interesting to understand how the discussion gets modified in this case.

${ }^{6}$ Recent results about the partition functions of seven-dimensional gauge theories on curved spaces $[73-$ 75] should have an M-theory interpretation in terms of the M-theory Hilbert space $\mathcal{H}_{M}\left(\partial \mathcal{M}_{11}\right)$ associated to this background.
} 
natural from the string theory point of view (and also for four dimensional theories with electromagnetic duality, although we will not consider such examples in this paper), so we will still adopt it.

Let us now discuss how to reproduce these results from the M-theory perspective, along the lines of [8]. The key fact is that in the presence of torsion at infinity the boundary values for the $F_{4}$ and $F_{7}$ fluxes do not commute [6-8]. The spaces in which we are engineering the seven dimensional $\mathfrak{g}_{\Gamma}$ theory are non-compact, so strictly speaking they have no boundary, but we will assume that whenever we have a space of the form $\mathcal{M}_{11}=\mathcal{M}_{p} \times \mathcal{C}\left(\mathcal{N}_{10-p}\right)$, with $\mathcal{C}\left(\mathcal{N}_{10-p}\right)$ asymptotically a cone over $\mathcal{N}_{10-p}$, then the right prescription for choosing asymptotic values for the fields is to quantize the theory on $\mathbb{R}_{t} \times \mathcal{M}_{10}$, with $\mathcal{M}_{10}:=$ $\mathcal{M}_{p} \times \mathcal{N}_{10-p}$, and to choose a state in the Hilbert space associated to $\mathcal{M}_{10}$. This prescription has appeared previously in the context of AdS/CFT [78, 79], and it was shown in [8] to lead to the right predictions in the context of six-dimensional SCFTs and $\mathcal{N}=4$ theories in four dimensions.

We are (thankfully) only interested in the grading of the Hilbert space of M-theory by topological class of the flux. In general, M-theory fluxes live on some cohomology theory $\mathbb{E}_{M}$, which is known not to be ordinary (singular, say) cohomology. A rather dramatic effect that can take us away from ordinary cohomology is that there is a shifted flux quantization condition [80]:

$$
\left[\frac{G_{4}}{2 \pi}\right]-\frac{p_{1}\left(\mathcal{M}_{11}\right)}{4} \in H^{4}\left(\mathcal{M}_{11} ; \mathbb{Z}\right)
$$

with $p_{1}\left(\mathcal{M}_{11}\right)$ the first Pontryagin class of the tangent bundle of $\mathcal{M}_{11}$. Fortunately this shifted quantization condition will not affect our discussion in any significant way, since $p_{1}\left(\mathcal{M}_{p} \times \mathcal{C}\left(\mathcal{N}_{10-p}\right)\right)=p_{1}\left(\mathcal{M}_{p}\right)+p_{1}\left(\mathcal{C}\left(\mathcal{N}_{10-p}\right)\right)$, which has legs either purely along $\mathcal{M}_{p}$ or $\mathcal{C}\left(\mathcal{N}_{10-p}\right)$. The fluxes of interest to us, on the other hand, have legs along both components. (An exception to this statement are fluxes associated with (-1)-form symmetries that we will encounter below, but in this paper we will not try to understand these in any detail.) Due to this fact we will use ordinary singular cohomology in our calculations below. ${ }^{7}$

The screening argument we discussed in the introduction gives the following defect group for the geometric engineering Hilbert space of this theory

$$
\mathbb{D}=Z\left(G_{\Gamma}\right)_{M 2}^{(1)} \times Z\left(G_{\Gamma}\right)_{M 5}^{(4)}
$$

Given flux operators $\Phi_{M 2, a}$ with $a \in$ Tor $H^{4}\left(\mathcal{M}_{10}\right)$ and $\Psi_{M 5, b}$ with $b \in \operatorname{Tor} H^{7}\left(\mathcal{M}_{10}\right)$ (measuring torsional M2 and M5 charge, respectively) we have that $[6,7]$

$$
\Phi_{M 2, a} \Psi_{M 5, b}=e^{2 \pi i \mathrm{~L}(a, b)} \Psi_{M 5, b} \Phi_{M 2, a}
$$

\footnotetext{
${ }^{7}$ The shifted quantization condition (2.1) is not necessarily the only issue with using singular cohomology, see for instance [81] for a discussion of further subtleties, and a proposal for a generalized cohomology theory taking them into account. The good agreement between the results from singular cohomology and field theory in the examples below encourages us to think that, at least in the simple backgrounds that we consider, our assumption for $\mathbb{E}_{M}$ is correct, but it would certainly be interesting to try and find sufficiently complicated backgrounds where singular cohomology is not enough, and understand the field theory implications of this fact.
} 
where

$$
\text { L: } \operatorname{Tor} H^{4}\left(\mathcal{M}_{10}\right) \times \operatorname{Tor} H^{7}\left(\mathcal{M}_{10}\right) \rightarrow \mathbb{Q} / \mathbb{Z}
$$

is the linking pairing in $\mathcal{M}_{10}$. The space $\mathbb{C}^{2} / \Gamma$ is a cone over $S^{3} / \Gamma$, and $\Gamma$ acts freely on the $S^{3}$, therefore in the case at hand $\mathcal{M}_{10}=\mathcal{M}_{7} \times\left(S^{3} / \Gamma\right)$. Assuming that $\mathcal{M}_{7}$ has no torsion we can apply the Künneth formula

$$
H^{n}\left(\mathcal{M}_{7} \times\left(S^{3} / \Gamma\right)\right)=\sum_{i+j=n} H^{i}\left(\mathcal{M}_{7}\right) \otimes H^{j}\left(S^{3} / \Gamma\right) .
$$

Since

$$
H^{\bullet}\left(S^{3} / \Gamma\right)=\left\{\mathbb{Z}, 0, \Gamma^{\mathrm{ab}}, \mathbb{Z}\right\}
$$

this implies that

$$
\text { Tor } H^{4}\left(\mathcal{M}_{10}\right)=H^{2}\left(\mathcal{M}_{7}\right) \otimes \Gamma^{\mathrm{ab}}=H^{2}\left(\mathcal{M}_{7} ; \Gamma^{\mathrm{ab}}\right)
$$

and

$$
\text { Tor } H^{7}\left(\mathcal{M}_{10}\right)=H^{5}\left(\mathcal{M}_{7}\right) \otimes \Gamma^{\mathrm{ab}}=H^{5}\left(\mathcal{M}_{7} ; \Gamma^{\mathrm{ab}}\right) .
$$

Writing, accordingly, $a=\alpha \otimes \ell_{a}$ and $b=\beta \otimes \ell_{b}$, with $\alpha \in H^{2}\left(\mathcal{M}_{7}\right), \beta \in H^{5}\left(\mathcal{M}_{7}\right)$ and $\ell_{i} \in H^{2}\left(S^{3} / \Gamma\right)=\Gamma^{\mathrm{ab}}$, we have

$$
\mathrm{L}(a, b)=(\alpha \cdot \beta) \mathrm{L}_{\Gamma}\left(\ell_{1}, \ell_{2}\right)
$$

with $\mathrm{L}_{\Gamma}$ the linking form in $S^{3} / \Gamma$. The general form for $\mathrm{L}_{\Gamma}$ is given in [82] - see table 1 . For instance consider the case $\Gamma=\mathbb{Z}_{N}$, corresponding to the $\mathfrak{s u}(N)$ theories in seven dimensions. We have $\Gamma^{\mathrm{ab}}=\left[\mathbb{Z}_{N}, \mathbb{Z}_{N}\right]=\mathbb{Z}_{N}$, with a linking form

$$
\mathrm{L}_{\Gamma}(1,1)=\frac{1}{N} \bmod 1
$$

This implies that the flux operators in this theory will not commute, indicating the defect group of this theory suffers from a mixed 't Hooft anomaly. In particular, this entails that some care is needed when choosing boundary conditions. Choose a basis of the Hilbert space that diagonalizes $\Phi_{a}$, for instance. ${ }^{8}$ Namely:

$$
\Phi_{a}|b\rangle=e^{2 \pi i \mathrm{~L}(a, b)}|b\rangle
$$

with $b \in$ Tor $H^{7}\left(\mathcal{M}_{10}\right)$. On the other hand, the states in this basis do not diagonalize $\Psi_{b}$

$$
\Psi_{b^{\prime}}|b\rangle=\left|b+b^{\prime}\right\rangle .
$$

There is analogously a basis of states $|a\rangle$, with $a \in \operatorname{Tor} H^{4}\left(\mathcal{M}_{10}\right)$ that diagonalizes the $\Psi_{b}$ operators. The two choices for the basis are related by a discrete Fourier transform:

$$
|a\rangle=\sum_{b} e^{2 \pi i \mathrm{~L}(a, b)}|b\rangle
$$

\footnotetext{
${ }^{8}$ That is, choose a basis for the Hilbert space which is the unique representation, by the Stone-von Neumanm theorem, of the Heisenberg group generated by the flux operators. We refer the reader to [83] for further background material on this topic, and to [69] for a clear illustration is a related context.
} 


\begin{tabular}{|cccc|}
\hline$\Gamma$ & $G_{\Gamma}$ & $\Gamma^{\mathrm{ab}}$ & $\mathrm{L}_{\Gamma}$ \\
\hline $\mathbb{Z}_{N}$ & $\mathrm{SU}(N)$ & $\mathbb{Z}_{N}$ & $\frac{1}{N}$ \\
$\operatorname{Dic}_{(4 N-2)}$ & $\operatorname{Spin}(8 N)$ & $\mathbb{Z}_{2} \oplus \mathbb{Z}_{2}$ & $\left(\begin{array}{cc}0 & 1 / 2 \\
1 / 2 & 0\end{array}\right)$ \\
$\operatorname{Dic}_{(4 N-1)}$ & $\operatorname{Spin}(8 N+2)$ & $\mathbb{Z}_{4}$ & $\frac{3}{4}$ \\
$\operatorname{Dic}_{(4 N)}$ & $\operatorname{Spin}(8 N+4)$ & $\mathbb{Z}_{2} \oplus \mathbb{Z}_{2}$ & $\left(\begin{array}{cc}1 / 2 & 0 \\
0 & 1 / 2\end{array}\right)$ \\
$\operatorname{Dic}_{(4 N+1)}$ & $\operatorname{Spin}(8 N+6)$ & $\mathbb{Z}_{4}$ & $\frac{1}{4}$ \\
$2 T$ & $E_{6}$ & $\mathbb{Z}_{3}$ & $\frac{2}{3}$ \\
$2 O$ & $E_{7}$ & $\mathbb{Z}_{2}$ & $\frac{1}{2}$ \\
$2 I$ & $E_{8}$ & 0 & 0 \\
\hline
\end{tabular}

Table 1. Linking pairings for $G_{\Gamma}$.

For instance, consider choosing a state $\left|0, L_{M 5}\right\rangle$ such that $\Psi_{b}\left|0, L_{M 5}\right\rangle=\left|0, L_{M 5}\right\rangle$ for all $b \in H^{7}\left(\mathcal{M}_{10}\right)$. This corresponds to setting all M5-brane fluxes to 0 , so that the M2 branes are genuine operators. In the seven dimensional theory we can interpret this choice as being in the $\mathrm{SU}(N)$ theory, with no background fluxes for the 1-form symmetry of this theory turned on, where the line operators coming from the wrapped M2 branes are genuine.

If, on the other hand, we choose our boundary conditions to be given by a state $\left|0, L_{M 2}\right\rangle$ such that $\Phi_{a}\left|0, L_{M 2}\right\rangle=\left|0, L_{M 2}\right\rangle$ for all $a \in H^{4}\left(\mathcal{M}_{10}\right)$, then our background will be in a superposition of all possible background fluxes for the M5-brane charge, since due to the properties of the Heisenberg algebra a change of basis from the electric to the magnetic basis is a discrete Fourier transform:

$$
\left|0, L_{M 2}\right\rangle=\sum_{a \in H^{4}\left(\mathcal{M}_{10}\right)}\left|a, L_{M 5}\right\rangle
$$

In terms of the seven dimensional theory, this implies being on a superposition of all possible values for the Stiefel-Whitney classes, or in other words choosing the $\mathrm{SU}(N) / \mathbb{Z}_{N}$ global form for the theory, having gauged the one-form symmetry of the $\mathrm{SU}(N)$ theory. Notice that, as a consequence of this gauging, the resulting theory has a magnetic $\mathbb{Z}_{N}^{(4)}$ higher symmetry.

\section{Higher form symmetries and non-commutative fluxes for 5d SCFTs}

The story is fairly similar for five dimensional theories engineered from M-theory on singular Calabi-Yau threefolds, but the possibilities in geometry and field theory are much richer. For concreteness, we will focus on geometries of the kind $\mathcal{M}_{5} \times \mathcal{V}_{6}$, where $\mathcal{M}_{5}$ is a closed Spin manifold without torsion, and $\mathcal{V}_{6}$ a non-compact Calabi-Yau threefold, given by the cone over some Sasaki-Einstein manifold $Y_{5}$. In order to make further progress below we 
will assume some additional conditions on $\mathcal{V}_{6}$, namely that $H_{2 n+1}\left(\mathcal{V}_{6}\right)=0$ for all $n$, and that Tor $H_{2 n}\left(\mathcal{V}_{6}\right)=0$ for all $n$. Toric Calabi-Yau threefold varieties are an important class of examples that satisfy these requirements, and we will focus mostly on these below (but the ideas generalize straightforwardly to other cases, such as cones over higher del Pezzo surfaces).

\subsection{Field theory analysis}

Let us first describe the expectations from field theory, in analogy with the sevendimensional discussion above. In the five dimensional theory we will have line and surface operators, which we will call Wilson lines and 't Hooft surfaces, following the standard terminology in the cases with a Lagrangian description. The charge of these objects is measured on three-dimensional and two-dimensional surfaces linking the respective objects in $\mathcal{M}_{5}$. Equivalently, depending on the global structure that we choose for the theory, we have 1-form symmetries with background fluxes valued on $H^{2}\left(\mathcal{M}_{5} ; \mathrm{Z}\right), 2$-form symmetries with background fluxes valued on $H^{3}\left(\mathcal{M}_{5} ; \mathbf{Z}\right)$, or combinations of both. Here $\mathbf{Z}$ is a group that in the cases with a Lagrangian is given by the subgroup of the universal cover of the gauge group that leaves all point operators invariant, as in [77]. For instance, if we have a 5 d Yang-Mills theory with algebra $\mathfrak{s u}(N)$ and matter in the adjoint, we have that $\mathbf{Z}=\mathbb{Z}_{N}$.

As in the seven dimensional case not all of these symmetries are simultaneously present in any given theory, and one cannot independently introduce background fluxes for all of them. Rather, we need to choose a maximal mutually local set of extended operators, and introduce fluxes only for those. In the Lagrangian context, this choice is a choice of the global form for the gauge group. For instance, if the algebra is $\mathfrak{s u}(N)$, a possible choice of global form is given by $\mathrm{SU}(N) / \mathbb{Z}_{N}$, where we sum over all background fluxes in $H^{2}\left(\mathcal{M}_{5} ; \mathbb{Z}_{N}\right)$ - that is, we sum over Stiefel-Whitney classes. Alternatively we could sum over bundles in $H^{3}\left(\mathcal{M}_{5} ; \mathbb{Z}_{2}\right)$. (As remarked above, the sum is not visible in the usual Lagrangian presentation.)

An interesting feature of five dimensional theories is that instanton configurations behave very much like particles. In the presence of a Chern-Simons coupling (for group theory reasons, this coupling is only available for $\mathfrak{s u}(N)$ with $N>2$ )

$$
S_{\mathrm{CS}}=k \int \Omega_{A}
$$

where $\Omega_{A}$ is the Chern-Simons form, these particles can potentially acquire a charge under the center of the gauge group. If this happens, then the higher form symmetry of the $\mathrm{SU}(N)$ theory can be (partially) broken, in the same way that ordinary matter in generic representations breaks the symmetry. Our task below will be to compute the charge of these particles under the center of the $\mathrm{SU}(N)$ gauge group, but the form of the coupling (3.1) suggests that the right answer will be that in the presence of such a coupling instanton particles acquire a charge $k$ under the $\mathbb{Z}_{N}$ center of the $\mathrm{SU}(N)$ theory. One heuristic way to argue for this is that an instanton background becomes, in the point-like limit $\operatorname{Tr}\left(F^{2}\right)=\delta^{4}(\vec{x})$, with $\vec{x}$ the directions transverse to the instanton, so the $\operatorname{Tr}\left(A \wedge F^{2}\right)$ term in $\Omega_{A}$ becomes an integral of $A$ over the worldline of the instanton particle, so the ChernSimons level $k$ can be identified with the charge of the particle. We will give below a 
more careful argument that shows that this is indeed the right result in the theories that we study. This implies that the center of a $\mathrm{SU}(N)$ theory at level $k$ is broken down to $\mathbb{Z}_{\operatorname{gcd}(N, k)}$ due to the charge of the instanton particles. ${ }^{9}$

The case of $\mathrm{SU}(2)$ is somewhat special: in this case $\Omega_{A}$ vanishes identically, but we can introduce a discrete $\theta$-term, given by 0 or 1 multiplied by the mod- 2 index (or equivalently, the $\eta$ invariant) of a fundamental fermion coupled to the $\mathrm{SU}(2)$ bundle that we are introducing. This coupling has the same effect as above: whenever the coefficient of the coupling is nonzero we find that the center of $\mathrm{SU}(2)$ is broken by the charge of instanton particles. (We will again give a detailed argument for this fact below.) This observation generalizes to the $\operatorname{USp}(2 N)$ theories: in these cases there is a nontrivial bordism group of Spin manifolds decorated with principal $U S p$ bundles, $\Omega_{5}^{\operatorname{Spin}}(B \operatorname{USp}(2 N))=\mathbb{Z}_{2}$ (see for instance [84] for a simple derivation). The non-trivial element in this bordism group can be detected by computing the $\eta$ invariant of a fermion in the fundamental representation, so one can couple a $\operatorname{USp}(2 N)$ theory to the SPT defined by this bordism invariant to introduce a discrete $\theta$ angle. Below we will only check explicitly that this coupling to a TQFT induces a charge under the $\mathbb{Z}_{2}$ center for instanton particles for $\mathrm{SU}(2)$, but this is actually enough to show that this must also happen for $\operatorname{USp}(2 N)$ with $N>1$, since in 5 dimensions every $\operatorname{USp}(2 N)$ bundle can be reduced to a $\operatorname{USp}(2)$ bundle, ${ }^{10}$ and the centers of $\operatorname{USp}(2 N)$ and any $\operatorname{USp}(2)$ subgroup can be identified canonically.

\subsection{M-theory and higher form symmetries}

We now want to understand the previous gauge theory discussion in terms of the Mtheory engineering of the relevant $5 \mathrm{~d}$ theories. The line and surface operators of the five dimensional operator will come from M2 and M5 branes wrapping suitable non-compact cycles in the internal toric Calabi-Yau threefold. As in the seven dimensional case, we classify which of these operators can be simultaneously taken to be genuine by looking to a maximal choice of commuting fluxes on the boundary $\partial \mathcal{M}_{11}$ (which, recall, has topology $\mathcal{M}_{5} \times Y_{5}$ in our case, with $Y_{5}$ a Sasaki-Einstein manifold). The non-trivial part of the flux commutation relations will come from the pairing

$$
\mathrm{L}: \operatorname{Tor} H^{4}\left(\partial \mathcal{M}_{11}\right) \times \operatorname{Tor} H^{7}\left(\partial \mathcal{M}_{11}\right) \rightarrow \mathbb{Q} / \mathbb{Z}
$$

We will show momentarily that $Y_{5}$ only has torsion in $H_{1}\left(Y_{5}\right)=H^{4}\left(Y_{5}\right)$ (or equivalently, by the universal coefficient theorem [86], in $H_{3}\left(Y_{5}\right)=H^{2}\left(Y_{5}\right)$ ). Together with the fact that $\partial \mathcal{M}_{11}=\mathcal{M}_{5} \times Y_{5}$, with $\mathcal{M}_{5}$ torsion-free, this implies that we can use the Künneth

\footnotetext{
${ }^{9}$ Denote by $p$ the order of the surviving group. By Lagrange's theorem $p$ divides $N$, so the subgroup is generated by $N / p$. For this element to leave a particle of charge $k$ invariant we need that $k N / p \equiv 0 \bmod$ $N$, or equivalently that $p$ divides $k$.

${ }^{10}$ The obstruction for reducing $\operatorname{USp}(2 N+2)$ bundles to $\operatorname{USp}(2 N)$ bundles in $d$ dimensions lives in $\pi_{i}(\mathrm{USp}(2 N+2) / \mathrm{USp}(2 N))$ for $i<d$ (see [85] for a review of the relevant facts for physicists). But $\mathrm{USp}(2 N+2) / \operatorname{USp}(2 N) \approx S^{4 N+3}$, so the obstructions to reducing to $\operatorname{USp}(2)$ vanish for $d<8$.
} 
formulas

$$
\begin{aligned}
& \text { Tor } H^{4}\left(\partial \mathcal{M}_{11}\right)=\left(H^{2}\left(\mathcal{M}_{5}\right) \otimes \operatorname{Tor} H^{2}\left(Y_{5}\right)\right) \oplus\left(H^{0}\left(\mathcal{M}_{5}\right) \otimes \operatorname{Tor} H^{4}\left(Y_{5}\right)\right) \\
& \text { Tor } H^{7}\left(\partial \mathcal{M}_{11}\right)=\left(H^{3}\left(\mathcal{M}_{5}\right) \otimes \text { Tor } H^{4}\left(Y_{5}\right)\right) \oplus\left(H^{5}\left(\mathcal{M}_{5}\right) \otimes \operatorname{Tor} H^{2}\left(Y_{5}\right)\right)
\end{aligned}
$$

Poincaré duality, together with the universal coefficient theorem, implies that Tor $H^{2}\left(Y_{5}\right)=$ Tor $H^{4}\left(Y_{5}\right)$. For conciseness, let us define

$$
\mathrm{Z}:=\text { Tor } H_{3}\left(Y_{5}\right)=\text { Tor } H^{2}\left(Y_{5}\right)=\text { Tor } H^{4}\left(Y_{5}\right)=\text { Tor } H_{1}\left(Y_{5}\right) .
$$

(In Lagrangian theories $\mathbf{Z}$ will be the center of the simply connected group with the given algebra.) The defect group for these geometries is

$$
\mathbb{D}=\left(\mathrm{Z}_{M 2}^{(1)} \oplus \mathrm{Z}_{M 5}^{(2)}\right) \oplus\left(\mathrm{Z}_{M 2}^{(-1)} \oplus \mathrm{Z}_{M 5}^{(4)}\right) .
$$

Under the assumption that $\mathcal{M}_{5}$ is torsion-free, the universal coefficient theorem implies that $H^{i}\left(\mathcal{M}_{5}\right) \otimes \mathbf{Z}=H^{i}\left(\mathcal{M}_{5} ; \mathbf{Z}\right)$, so the first terms on the right hand side are the ones we had anticipated from our field theory analysis above. We see that in the M-theory language these cohomology groups parametrize the flux operators measuring the fluxes created by M2 branes wrapping non-compact two-cycles in $V$, and M5 branes wrapping non-compact four-cycles in $V$, respectively, as one would have expected. The last two terms correspond to $(-1)$-form and 4 -form symmetries, which are somewhat more exotic from the field theory point of view, and we will ignore them in our analysis. (See [87-89] for recent work exploring such symmetries from the field theory point of view.)

For our purposes it will be convenient to work with a smoothed out and compactified version of the geometry. Denote by $\tilde{\mathcal{V}}_{6}$ some smooth crepant resolution of $\mathcal{V}_{6}$ (it does not matter which one), and introduce $X_{6}:=B_{6} \cap \tilde{\mathcal{V}}_{6}$, where $B_{6}$ is a sufficiently large ball containing the exceptional set of $\tilde{\mathcal{V}}_{6}$. Branes extending from the origin to infinity in $\mathcal{V}_{6}$ will map to branes extending to the boundary of $X_{6}$. We have that $H_{i}\left(\tilde{\mathcal{V}}_{6}\right)=H_{i}\left(X_{6}\right)$, and since (topologically) $Y_{5}=\partial X_{6}$ we have a long exact sequence of the form

$$
\ldots \rightarrow H_{n}\left(Y_{5}\right) \rightarrow H_{n}\left(X_{6}\right) \rightarrow H_{n}\left(X_{6}, Y_{5}\right) \rightarrow H_{n-1}\left(Y_{5}\right) \rightarrow \ldots
$$

The physical interpretation of this long exact sequence in our physical context is as follows: branes wrapped on $H_{n}\left(X_{6}\right)$ give rise to dynamical objects in the theory. Branes going to infinity will be associated to elements of the relative homology groups $H_{n}\left(X_{6}, Y_{5}\right)$ : these are defined to be those cycles in $X_{6}$ that are closed, modulo cycles living on $Y_{5}$. These branes wrapping non-compact cycles give rise to defects in the theory, whose lattice of charges is given precisely by this group. However, physically, we are only interested in the charges that survive 't Hooft screening by dynamical operators, mathematically this is encoded in the fact that we only care about the quotient $H_{n}\left(X_{6}, Y_{5}\right) / H_{n}\left(X_{6}\right)$, or equivalently that we only need to know about the homology class of the intersection of the non-compact cycle with the boundary, namely $H_{n-1}\left(Y_{5}\right)$. (The long exact sequence does not necessarily 
truncate on $H_{n-1}\left(Y_{5}\right)$ in general, so this statement would need correction in those cases in which it doesn't, but the truncation does take place in all cases of interest to us.)

Next, we use Lefschetz duality [86] to rewrite

$$
H_{k}\left(X_{6}, Y_{5}\right)=H_{c}^{n-k}\left(X_{6}\right) .
$$

We will assume that $X_{6}$ is torsion free (which is true, in particular, for toric varieties [9092]), so by the universal coefficient theorem for cohomology we get

$$
H_{k}\left(X_{6}, Y_{5}\right)=H_{c}^{n-k}\left(X_{6}\right)=\operatorname{Hom}\left(H_{n-k}\left(X_{6}\right), \mathbb{Z}\right) .
$$

If we now assume that $H_{2 n+1}\left(X_{6}\right)=0$ (which is again true in the special case of toric varieties [90-92], but also more generally), the long exact sequence (3.6) reduces to

$$
\begin{aligned}
& 0 \rightarrow \operatorname{Hom}\left(H_{0}\left(X_{6}\right), \mathbb{Z}\right) \rightarrow H_{5}\left(Y_{5}\right) \rightarrow 0, \\
& 0 \rightarrow H_{4}\left(Y_{5}\right) \rightarrow H_{4}\left(X_{6}\right) \stackrel{Q_{4}}{\longrightarrow} \operatorname{Hom}\left(H_{2}\left(X_{6}\right), \mathbb{Z}\right) \stackrel{\partial_{4}}{\longrightarrow} H_{3}\left(Y_{5}\right) \rightarrow 0, \\
& 0 \rightarrow H_{2}\left(Y_{5}\right) \rightarrow H_{2}\left(X_{6}\right) \stackrel{Q_{2}}{\longrightarrow} \operatorname{Hom}\left(H_{4}\left(X_{6}\right), \mathbb{Z}\right) \stackrel{\partial_{2}}{\longrightarrow} H_{1}\left(Y_{5}\right) \rightarrow 0, \\
& 0 \rightarrow H_{0}\left(Y_{5}\right) \rightarrow H_{0}\left(X_{6}\right) \rightarrow 0,
\end{aligned}
$$

where, the homomorphisms $Q_{k}: H_{k}\left(X_{6}\right) \rightarrow \operatorname{Hom}\left(H_{6-k}\left(X_{6}\right), \mathbb{Z}\right)$ are given by partial evaluation of the intersection forms

$$
q_{k}: H_{k}\left(X_{6}\right) \times H_{6-k}\left(X_{6}\right) \rightarrow \mathbb{Z}
$$

with $k=2,4$. That is, $Q_{k}(x)(y)=q_{k}(x, y)$. Note that $Q_{4}=Q_{2}^{t}$. It follows from these exact sequences that $H_{0}\left(Y_{5}\right)=H_{5}\left(Y_{5}\right)=H_{0}(X)=\mathbb{Z}$, and that

$$
\begin{array}{ll}
H_{4}\left(Y_{5}\right)=\operatorname{ker}\left(Q_{4}\right), & H_{3}\left(Y_{5}\right)=\operatorname{coker}\left(Q_{4}\right), \\
H_{2}\left(Y_{5}\right)=\operatorname{ker}\left(Q_{2}\right), & H_{1}\left(Y_{5}\right)=\operatorname{coker}\left(Q_{2}\right),
\end{array}
$$

so finally

$$
\mathbf{Z}=\text { Tor coker }\left(Q_{4}\right)=\text { Tor coker }\left(Q_{2}\right) .
$$

Having understood the space of charge operators for the five dimensional theory, we still need to find their commutation relations. This follows straightforwardly from (2.3), in a way very analogous to (2.9). Writing $a=\alpha \otimes \Sigma_{a}$ and $b=\beta \otimes D_{b}$, with $\alpha \in H^{2}\left(\mathcal{M}_{5}\right)$, $\beta \in H^{3}\left(\mathcal{M}_{5}\right), \Sigma_{a} \in$ Tor $H^{2}\left(Y_{5}\right)$ and $D_{b} \in$ Tor $H^{4}\left(Y_{5}\right)$, we have

$$
\mathrm{L}(a, b)=(\alpha \cdot \beta) \mathrm{L}_{Y_{5}}\left(\Sigma_{a}, D_{b}\right)
$$

with $\mathrm{L}_{Y_{5}}$ the linking form in $Y_{5}$, a perfect pairing

$$
\mathrm{L}_{Y_{5}}: \operatorname{Tor} H^{k}\left(Y_{5}\right) \times \operatorname{Tor} H^{5-k+1}\left(Y_{5}\right) \rightarrow \mathbb{Q} / \mathbb{Z} .
$$

We can derive $L_{Y_{5}}$ from knowledge of the intersection matrix $Q_{4}=Q_{2}^{t}$ as follows. Let $\sigma \in$ Tor $H_{3}\left(Y_{5}\right)$ and $\bar{\sigma} \in$ Tor $H_{1}\left(Y_{5}\right)$, and choose $\mu \in \operatorname{Hom}\left(H_{2}\left(Y_{5}\right), \mathbb{Z}\right)$ and $\bar{\mu} \in \operatorname{Hom}\left(H_{4}\left(Y_{5}\right), \mathbb{Z}\right)$ such that, $\partial_{4} \mu=\sigma$ and $\partial_{2} \bar{\mu}=\bar{\sigma}$. Then, for non-trivial Tor $H_{3}\left(Y_{5}\right)$ and Tor $H_{1}\left(Y_{5}\right)$, there 
are non-zero integers $n$ and $m$ such that $\partial(n \mu)=n \sigma=0$ and $\bar{\partial}(m \bar{\mu})=m \bar{\sigma}=0$. Thus, we may pick $\nu \in H_{4}\left(X_{6}\right)$ and $\bar{\nu} \in H_{2}\left(X_{6}\right)$ such that, $Q_{4} \nu=n \mu$ and $Q_{2} \bar{\nu}=m \bar{\mu}$. The linking pairing is then ${ }^{11}[93]$

$$
\mathrm{L}_{Y_{5}}(\sigma, \bar{\sigma}) \equiv \frac{1}{n m} q(\nu, \bar{\nu}) \quad \bmod 1
$$

This may be equivalently written as

$$
\mathrm{L}_{Y_{5}}(\sigma, \bar{\sigma}) \equiv q^{-1}(\mu, \bar{\mu}) \bmod 1,
$$

where $q^{-1}: \operatorname{Hom}\left(H_{2}\left(X_{6}\right), \mathbb{Z}\right) \times \operatorname{Hom}\left(H_{4}\left(X_{6}\right), \mathbb{Z}\right) \rightarrow \mathbb{Q}$. More explicitly, this means that, if $\alpha_{i}^{\prime *}$ is a generator of $\operatorname{Hom}\left(H_{2}\left(X_{6}\right), \mathbb{Z}\right)$ and $\beta_{j}^{\prime *}$ is a generator of $\operatorname{Hom}\left(H_{4}\left(X_{6}\right), \mathbb{Z}\right)$ such that $\partial \alpha_{i}^{*}$ is the generator of Tor $H_{3}\left(Y_{5}\right)$ and $\bar{\partial} \beta_{j}^{\prime *}$ is the generator of Tor $H_{1}\left(Y_{5}\right)$ then, the linking number is just the $(i \times j)$ th element of $q^{-1}$ :

$$
\mathrm{L}_{Y_{5}}\left(\partial \alpha_{i}^{\prime *}, \bar{\partial} \beta_{j}^{*}\right)=q^{-1}\left(\alpha_{i}^{\prime *}, \beta_{j}^{*}\right)=q_{i j}^{-1} \bmod 1 .
$$

The appendices contain various worked out examples of the application of this relation, which encodes the discrete mixed 't Hooft anomaly coefficients for defect groups associated to the higher form symmetries of 5d SCFTs.

\subsection{The case of toric Calabi-Yau varieties}

An important special case of the previous discussion is that when $\mathcal{V}_{6}$ is a toric Calabi-Yau variety. (We refer the reader to [94-97] for systematic reviews of toric geometry.)

The crepant resolution $\tilde{\mathcal{V}}_{6}$ is obtained by choosing a triangulation for the toric diagram. As mentioned above, the odd dimensional homology groups of a toric variety vanish, and the even homology groups for $X_{6}$ can be easily obtained by looking at the toric diagram for $\mathcal{V}_{6}$. Let $I$ be the number of points in the interior of the diagram and $B$ be the number of points on the edges of the diagram. Then the number of 4-cycles is $I$, and since $\mathcal{V}_{6}$ is connected the number of 0 -cycles is 1 . The Euler characteristic of the Calabi-Yau equals to the number of 2-dimensional faces of the resolved toric diagram [98], which is twice the area $A$ of the toric diagram. The Euler characteristic also equals the number of even-dimensional cycles minus the number of odd-dimensional cycles. We know by Pick's theorem that $2 A=2 I+B-2$, so the number of 2 -cycles is $I+B-3$, so we have

$$
H_{0}(X)=\mathbb{Z}, H_{2}(X)=\mathbb{Z}^{(I+B-3)}, H_{4}(X)=\mathbb{Z}^{I}, H_{6}(X)=0 .
$$

One can also compute $Q_{4}$ (or equivalently $Q_{2}$ ) in toric varieties quite conveniently purely in terms of the toric data. What one needs to construct is the Mori cone of effective curves in the toric variety, and find their intersections with the compact divisors, which are manifest in the toric description as points in the interior of the toric diagram. Well developed algorithms for doing this exist, reviewed for example in [95], and implemented for instance in SAgE [99]. As an example, consider the Calabi-Yau cone over $\mathbb{F}_{0}=\mathbb{P}^{1} \times \mathbb{P}^{1}$. This geometry can be alternatively described as the (real) Calabi-Yau cone over $Y^{2,0}$. Its

\footnotetext{
${ }^{11}$ Note that we use $q$ and $q^{t}$ interchangeably. It should be clear from the context which one we mean.
} 
toric diagram has external vertices $p_{i}=\{(1,0),(-1,0),(0,1),(0,-1)\}$, and an internal vertex at $t=(0,0)$. Its Mori cone is generated by two curves $\mathcal{C}_{1}, \mathcal{C}_{2}$ with intersection matrix with the toric divisors given by

\begin{tabular}{c|ccccc} 
& $V\left(p_{1}\right)$ & $V\left(p_{2}\right)$ & $V\left(p_{3}\right)$ & $V\left(p_{4}\right)$ & $V(t)$ \\
\hline $\mathcal{C}_{1}$ & 1 & 0 & 1 & 0 & -2 \\
$\mathcal{C}_{1}$ & 0 & 1 & 0 & 1 & -2
\end{tabular}

We have included all toric divisors here, but the $V\left(p_{i}\right)$ divisors are non-compact. The divisor $V(t)$ is compact, on the other hand, so we find that

$$
Q_{2}=(-2-2)
$$

and we predict that

$$
\mathrm{Z}=\operatorname{Tor} \operatorname{coker}\left(Q_{2}\right)=\mathbb{Z}_{2} .
$$

We will see below that this result agrees with the expectation from field theory: the defect group in this case is

$$
\mathbb{D}=\mathbb{Z}_{2}^{(1)} \oplus \mathbb{Z}_{2}^{(2)}
$$

All the results below can be derived using these methods, but in practice it is much more efficient to use instead a method introduced (to our knowledge) in [100], which avoids the need to introduce a triangulation or computing the Mori cone. Consider a toric CalabiYau cone with an isolated singularity, and $v$ external vertices. (In section 5.2 we will derive a modified form of the method in [100] valid for non-isolated singularities.) In terms of the toric diagram, this means that there are no lattice points along the edges of the toric diagram. As argued in [100], one has that

$$
H_{i}\left(Y_{5}\right)=H_{i}\left(B_{3}^{L}\right) \quad \text { for } i \leq 2,
$$

where $B_{3}^{L}$ is a chain of lens spaces $L_{n_{1}}, \ldots, L_{n_{v}}$, joined at their torsion cycle, constructed as follows. For each external vertex $p_{i}, i \in\{1, \ldots, v\}$, construct the triangle $T_{i}$ defined by the vertex and the two vertices adjacent to it, that is, the convex hull of $\left\{p_{i-1}, p_{i}, p_{i+1}\right\}$ (with $p_{0}:=p_{v}$ and $\left.p_{v+1}:=p_{1}\right)$. Then $n_{i}=2 \operatorname{Area}\left(T_{i}\right)$. Additionally, one can show that $[100]$

$$
H_{1}\left(B_{3}^{L}\right)=\mathbb{Z}_{\operatorname{gcd}\left(n_{1}, \ldots, n_{v}\right)},
$$

so we find that in the toric case

$$
\mathrm{Z}=\mathbb{Z}_{\mathrm{gcd}\left(n_{1}, \ldots, n_{v}\right)}
$$

Coming back to our $\mathcal{C}_{\mathbb{R}}\left(Y^{2,0}\right)$ example, we have four triangles, all of unit area. So

$$
\mathrm{Z}=\mathbb{Z}_{\mathrm{gcd}(2,2,2,2)}=\mathbb{Z}_{2}
$$

\section{$4 \mathfrak{s u}(p)_{k}$ theory}

We will now apply the previous results to a simple set of cases: $\mathfrak{s u}(p)$ theories at ChernSimons level $k$. 

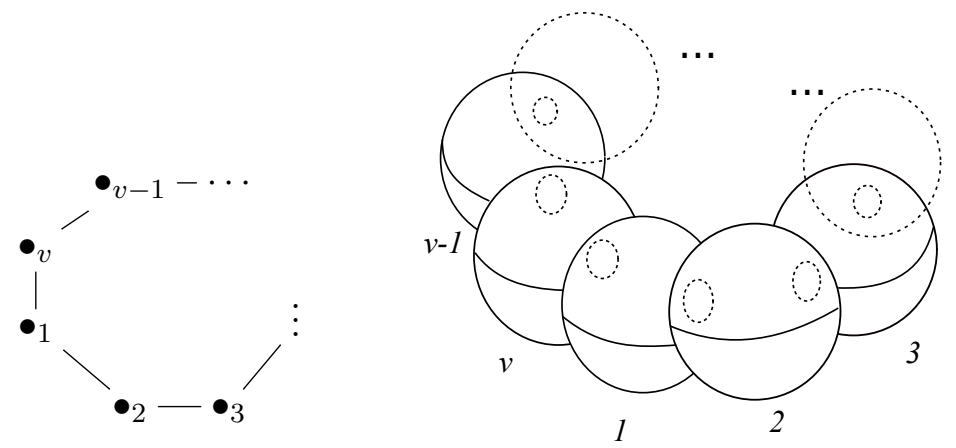

Figure 1. Schematic topology of $B_{3}^{L}[100]$.

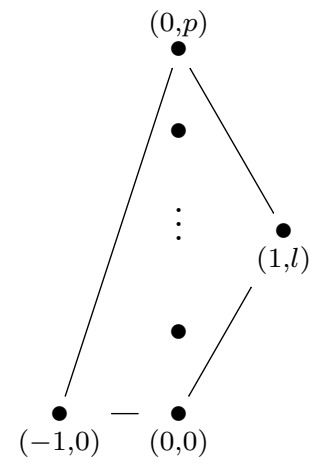

Figure 2. Toric diagram for $Y^{p, q}$. We have defined $l \equiv p-q$.

\subsection{Toric realization and geometric computation}

It is well-known that $\mathfrak{s u}(p)_{k}$ theories can be obtained exploiting canonical CY singularities that are cones over Sasaki-Einstein manifolds of $Y^{p, q}$ type (see, for instance, [41] for a recent account and [101] for the original analysis of these geometries). Let us introduce for convenience $q:=-k$, and we will assume $0 \leq q<p$. We show the resulting toric diagram in figure 2.

From our general discussion above, we need to compute Tor $H^{2}\left(Y^{p, q}\right)=$ Tor $H^{4}\left(Y^{p, q}\right)$, together with the linking pairing, in order to determine the Heisenberg group encoding the higher symmetries of the theory. Whenever $p$ and $q$ are relatively prime, we have that [101] $Y^{p, q}$ is topologically $S^{2} \times S^{3}$, so there is no torsion. So in these cases there is no choice of global structure for the field theory. More interesting is the case where $\operatorname{gcd}(p, q) \neq 1$. We can compute the relevant torsion groups following the general prescription in section 3.3 as follows. Choose an ordering of the external points of the toric diagram in figure 2 such that adjacent points are consecutive. For instance, choose

$$
\left\{p_{1}, p_{2}, p_{3}, p_{4}\right\}=\{(-1,0),(0,0),(1, l),(p, 0)\}
$$

Define now the triangles $T_{i}, i \in\{1, \ldots, 4\}$, as the convex hull of $\left\{p_{i-1}, p_{i}, p_{i+1}\right\}$ (with 


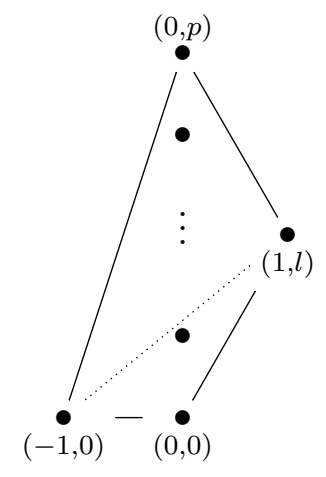

Figure 3. One of the triangles defined in the text.

$p_{0}:=p_{4}$ and $\left.p_{5}:=p_{1}\right)$. We show the triangle $T_{2}$ in figure 3 as an example. We have that

$$
\text { Tor } H^{2}\left(Y^{p, q}\right)=\text { Tor } H^{4}\left(Y^{p, q}\right)=\mathbb{Z}_{\operatorname{gcd}\left(n_{1}, n_{2}, n_{3}, n_{4}\right)}
$$

where $n_{i}$ is defined as twice the area of $T_{i}$. It is elementary to show that $n_{i}=\{p, l, p, 2 p-$ $l\}=\{p, p-q, p, p+q\}$, which implies that

$$
\mathrm{Z}=\text { Tor } H^{2}\left(Y^{p, q}\right)=\operatorname{Tor} H^{4}\left(Y^{p, q}\right)=\mathbb{Z}_{\mathrm{gcd}(p, q)} .
$$

We show in appendix B.1 that the linking pairing $\mathrm{L}_{Y^{p, q}}$ : Tor $H^{2}\left(Y^{p, q}\right) \times \operatorname{Tor} H^{4}\left(Y^{p, q}\right) \rightarrow$ $\mathbb{Q} / \mathbb{Z}$ is

$$
\mathrm{L}_{Y^{p, q}}(1,1)=-\frac{1}{\operatorname{gcd}(p, q)} \bmod 1 .
$$

In the case that the Chern-Simons level $k$ vanishes this leads to $Z=\mathbb{Z}_{p}$, which is the expected result for pure $\mathcal{N}=1 \mathfrak{s u}(p)_{0}$ theory in five dimensions. This theory admits a number of global variants, for instance $\mathrm{SU}(p)_{0}$ or $\operatorname{PSU}(p)_{0}:=\mathrm{SU}(p)_{0} / \mathbb{Z}_{p}$. The classification of all such global forms proceeds just as in the case of $\mathfrak{s u}(p)$ theories in four dimensions [77], so we will not delve on it further. The case with $k \neq 0$ is more subtle, and we turn to it now.

\subsection{Non-vanishing Chern-Simons levels and the charge of instanton particles}

We would now like to reproduce the M-theory results above from a field theoretical perspective, particularly in the case $q>0$. We will see that the instanton particles are charged under the center $\mathbb{Z}_{p}$ of the gauge group, so they will break this group to the subgroup under which the instanton particles are uncharged.

In order to determine the charge of the instanton particles, note that the instanton particles will arise in M-theory from M2 branes wrapping holomorphic curves in the geometry. On the other hand, the center of the gauge group lies in the orbit of the Cartan generators, which can be understood geometrically in terms of divisors in the M-theory picture. So, in this context, the charge of the instanton particles will be encoded in an intersection of the effective curve that the instanton is wrapping with some suitably chosen combination of divisors. 


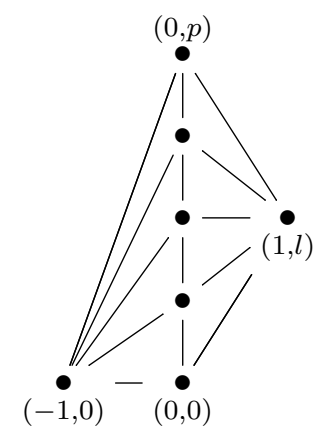

Figure 4. Triangulation of $Y^{p, q}$ considered in the text.

It is useful to choose the triangulation of the $\mathcal{C}\left(Y^{p, q}\right)$ geometry given in figure 4 . Denoting the points at $(0, i)$ by $I_{i}$, the cones of this triangulation are of the form $\left(p_{1}, I_{i}, I_{i+1}\right)$ and $\left(p_{3}, I_{i}, I_{i+1}\right)$. The instanton particles are naturally associated to the curves $\mathcal{C}_{i}:=$ $D_{I_{i}} \cdot D_{I_{i-1}}$, with $D_{I_{i}}$ the toric divisor associated to the point $I_{i}$, and $i \in\{1, \ldots, p\}$. On the other hand, the Cartan generators are associated to the interior points: for each compact four-cycle $D$ in the geometry we have a Poincaré dual harmonic two-form $\operatorname{PD}\left[D_{i}\right]$, and dimensional reduction of $C_{3}$ along these harmonic forms gives rise to the gauge bosons in the Cartan of the five dimensional theory. In the geometry at hand the compact four-cycles are generated by the toric divisors associated to the interior points $I_{i}, i \in\{1, \ldots, p-1\}$. The charge of a curve $\mathcal{C}$ under the $\mathrm{U}(1)$ Cartan associated to the divisor is then simply

$$
Q_{i}(\mathcal{C})=\int_{\mathcal{C}} \operatorname{PD}\left[D_{I_{i}}\right]=D_{I_{i}} \cdot \mathcal{C}
$$

We are only interested on a very specific element of the Cartan of $\mathrm{SU}(p)$, the generator of the $\mathbb{Z}_{p}$ center of the group. This generator is of the form

$$
g_{Z}=\left(\begin{array}{llll}
\omega_{p} & & & \\
& \omega_{p} & & \\
& & \ddots & \\
& & & \omega_{p}
\end{array}\right)
$$

with $\omega_{p}=\exp (2 \pi i / p)$. On the other hand, it is natural to choose an embedding of the $\mathrm{U}(1)$ subgroups associated to the $D_{i}$ into $\mathrm{SU}(p)$ of the form

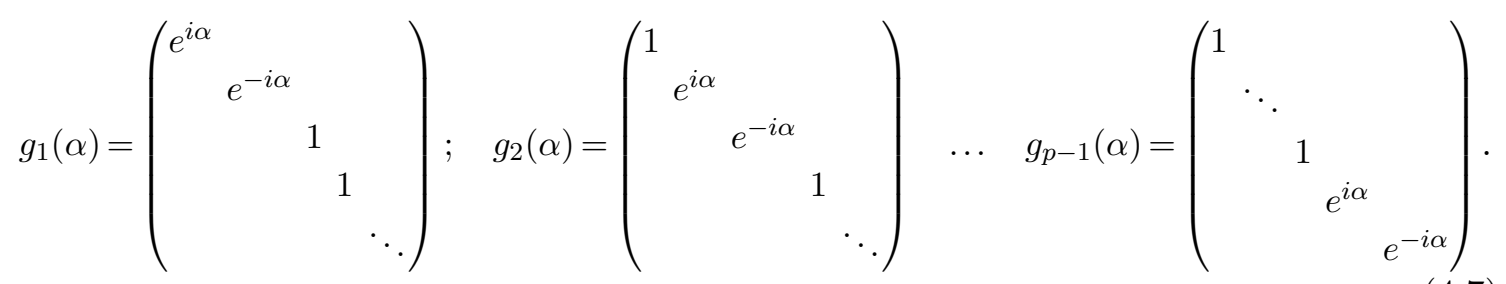

(One reason that this embedding is natural is that the $D_{I_{i}}$ geometrically parametrize separation of the Cartan branes in a $\mathrm{U}(p)$ stack, and each Cartan brane in the $\mathrm{U}(p)$ theory 
naturally embed as $\operatorname{diag}\left(1, \ldots, 1, e^{i \alpha}, 1, \ldots\right)$.) We can write

$$
g_{Z}=\prod_{k=1}^{p-1}\left(g_{k}(2 \pi / p)\right)^{k} .
$$

So if we want to measure the charge of a curve $\mathcal{C}$ under the generator $g_{Z}$ of the center of $\mathrm{SU}(p)$, we can define a divisor $Z:=\sum_{k=1}^{p-1} k D_{I_{k}}$, and the charge will be given by

$$
Q_{Z}(\mathcal{C}) \equiv \mathcal{C} \cdot Z=\sum_{k=1}^{p-1} k \mathcal{C} \cdot D_{I_{k}} \quad \bmod p
$$

where we have used the fact that the charge under the centre is defined modulo $p$.

We thus only need to determine the charge of the instanton particles associated to $\mathcal{C}_{j}$ under the Cartan associated to $D_{I_{i}}$. We denote this by $Q_{i}\left(\mathcal{C}_{j}\right)$. It is straightforward to compute from the toric data, and it was also obtained using field theory methods in [41]. Either way, one obtains

$$
\begin{aligned}
Q_{i-1}\left(\mathcal{C}_{i}\right) & =(p-q-2 i) \\
Q_{i}\left(\mathcal{C}_{i}\right) & =-(p-q-2(i-1))
\end{aligned}
$$

with all other charges vanishing. The charge under the centre is thus

$$
Q_{Z}\left(\mathcal{C}_{i}\right) \equiv(i-1)(p-q-2 i)-i(p-q-2(i-1)) \equiv q \bmod p .
$$

So we obtain the result (that one might have guessed from the form of the Chern-Simons coupling in the first place) that instanton particles have charge $q$ under the center of $\mathrm{SU}(p)$. Recalling that the subgroup of $\mathbb{Z}_{p}$ preserved by a particle with charge $q$ is precisely $\operatorname{gcd}(p, q)$, we reproduce the result from the geometric computation above.

There is one last remaining subtlety to take care of: we have just shown that there are instantonic particles have charge $q \bmod p$, but it could in principle be possible that there is some class of particles with charge different from $q$ or 0 under $\mathbb{Z}_{p}$, which would change the result. It is not difficult to show that this is not the case, as follows. Every particle will wrap an effective curve in the geometry, or in other words a curve in the Mori cone of the toric variety. This Mori cone is finitely generated, we give a set of generators in appendix A. It is a simple exercise, using the ideas above, to show that all the generators of the Mori cone found there have charge 0 or $q$ under $\mathbb{Z}_{p}$, so the same will hold for any curve in the Mori cone. So we can conclude that the $\mathbb{Z}_{\operatorname{gcd}(p, q)} 1$-form symmetry is not broken any further by M2 brane states.

\subsection{Adding fundamental matter}

As a final check of our formalism, let us consider the case of the $\operatorname{SU}(p)_{q}$ theory with a hypermultiplet in the fundamental representation. This theory can be engineered by considering the geometry in figure 5 [102]. It is related by adding a triangle to the $Y^{p, q}$ toric diagram. Note that the added triangle is of minimal area, which implies that there is no torsion in the horizon manifold for this geometry, so we will have that $Z$ is trivial, reproducing the field theory expectation that upon addition of a flavor in the fundamental representation the higher form symmetry is broken. 


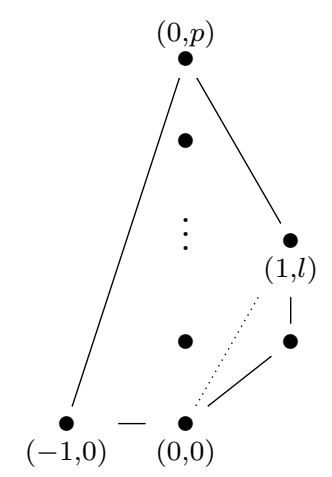

Figure 5. Geometry realizing $\mathfrak{s u}(p)$ with one flavor. We show the added triangle, which is of minimal area.

\section{$5 \quad$ Higher form symmetries and 5d duality}

Often massive deformations of a 5d SCFTs can give rise to gauge theory phases [16]. A given 5d SCFT can admit several such phases related to inequivalent effective gauge theories thus resulting in a so called 5d "duality" $[19,102]$ (see also [45] for a huge list of novel such dualities predicted using geometric engineering). Here we use the word duality in quotes to emphasize that this is not a duality in any conventional field theoretical sense, rather the manifestation that the Coulomb phases of certain effective gauge theory description of a relevant deformation of a 5d SCFT happen to overlap [41]. Higher form symmetries provide an interesting consistency condition: these deformations are neutral with respect to the higher forms of the SCFTs, and therefore different $5 \mathrm{~d}$ dual gauge theories must have the same higher form symmetries.

In this section we illustrate some examples of this consistency checks building upon a field theory analysis. As many of these examples have also nontrivial nonabelian 0-form symmetry groups, the corresponding geometries typically have corresponding curves of singularities, and our prescription to compute the center symmetry must be modified to include non-isolated singularities. Having at our disposal several gauge theory examples, it is easy to conjecture a modified prescription that works in this case and reproduces all the field theoretical results. After motivating the conjecture from physical considerations, we will prove that it is indeed correct.

\subsection{The beetles}

Consider the 5 d duality

$$
\mathrm{SU}(2)_{\pi} \times \mathrm{SU}(2)_{\pi} \longleftrightarrow \mathrm{SU}(3)_{0} N_{f}=2
$$

which can be understood from the corresponding geometry as follows. The 5d SCFT from which this $5 \mathrm{~d}$ duality originates is realized as a $\mathcal{T}_{\mathcal{V}_{6}}$ where $\mathcal{V}_{6}$ is the toric $\mathrm{CY}$ singularity 
with toric diagram

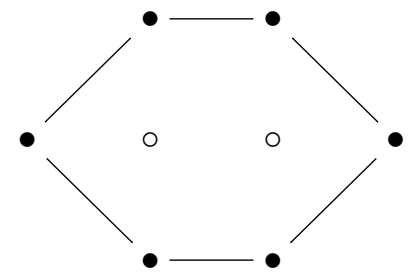

the so called 'beetle geometry' [41]. The $\mathrm{SU}(2) \times \mathrm{SU}(2)$ phase can be characterized via the vertical reduction/ruling (see [18] for the original analysis and [41] for some recent results)
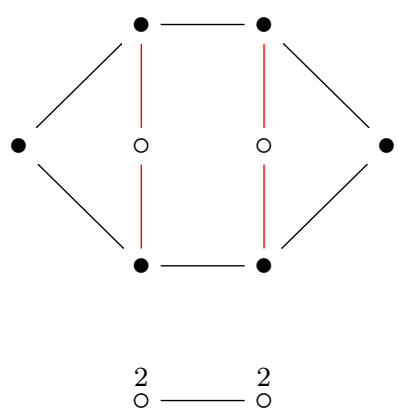

The $\mathrm{SU}(3) N_{f}=2$ phase can be characterized via the horizontal reduction/ruling
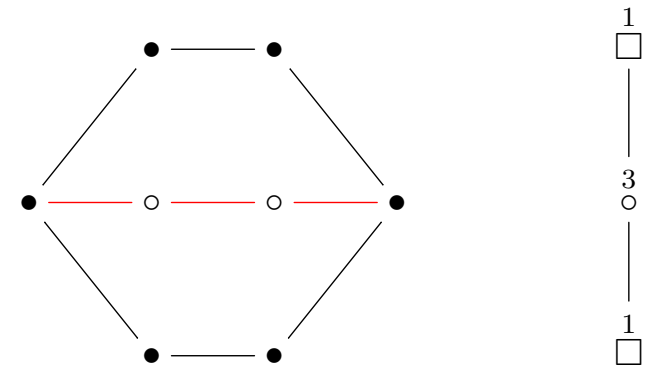

From this latter perspective it is clear that this model cannot have higher form symmetries: the fundamental of $\mathrm{SU}(3)$ has unit charge with respect to the center of the gauge group, and therefore the corresponding one form symmetry is broken. However, from the perspective of the $\mathrm{SU}(2) \times \mathrm{SU}(2)$ phase, it might seem that we still have a one form symmetry: indeed via the isomorphism

$$
\mathrm{SU}(2) \times \mathrm{SU}(2) \simeq \mathrm{Spin}(4)
$$

we can identify the bifundamental $(\mathbf{2}, \overline{\mathbf{2}})$ as a $\mathbf{4}_{v}$, which has charge $(1,0)$ with respect to the $\mathbb{Z}_{2} \times \mathbb{Z}_{2}$ center of Spin(4). For this reason one might expect to have a surviving $\mathbb{Z}_{2}$ factor. The solution of this conundrum is given by realizing that we have a non-trivial discrete theta term for the two $\mathrm{SU}(2)$ factors in this theory. Geometry clearly encodes this fact: recall that the singularity obtained by shrinking a $d P_{1}$ surface to zero size corresponds to the $\mathrm{SU}(2)_{\pi}$ gauge theory, that has a nontrivial discrete theta angle (and no one form symmetry, as we showed in section 4). Cutting the toric diagram open along the dotted 
diagonal
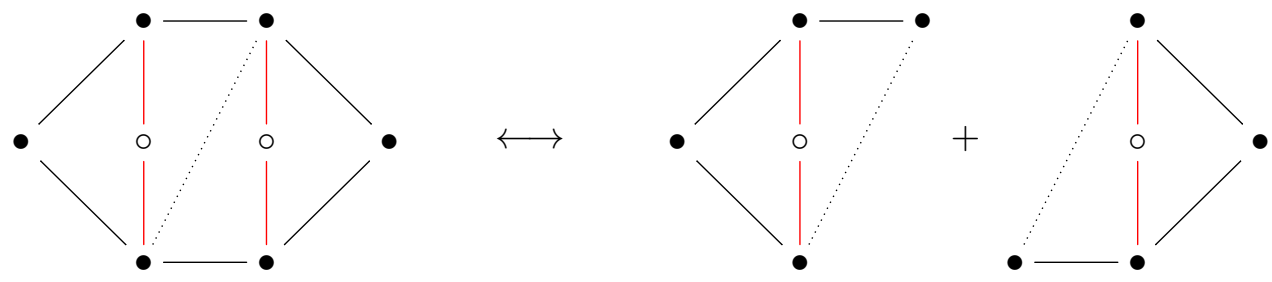

we clearly see that we can identify the two gauge theory subsectors with $\mathrm{SU}(2)_{\pi}$ theories that have a trivial $\mathbb{Z}_{2}$ one-form symmetry: because of the discrete theta term, the corresponding BPS instantons are charged with respect to the center of the gauge group and break the diagonal $\mathbb{Z}_{2}$. This result clearly matches our prescription: the defect group for this geometry is trivial, since this geometry has outer triangles of minimal area.

This argument generalizes to the case of other quiver theories. As an example let's consider the duality

$$
\mathrm{SU}(2)_{\pi} \times \underbrace{\mathrm{SU}(2)_{0} \times \cdot \times \mathrm{SU}(2)_{0}}_{N-2 \text { times }} \times \mathrm{SU}(2)_{\pi} \quad \longleftrightarrow \quad \mathrm{SU}(N+1) N_{f}=2 N-2 .
$$

The $5 \mathrm{~d}$ SCFT from which this 5 d duality originates is realized as a $\mathcal{T}_{\mathcal{V}_{6}}$ where $\mathcal{V}_{6}$ is the toric CY singularity with toric diagram

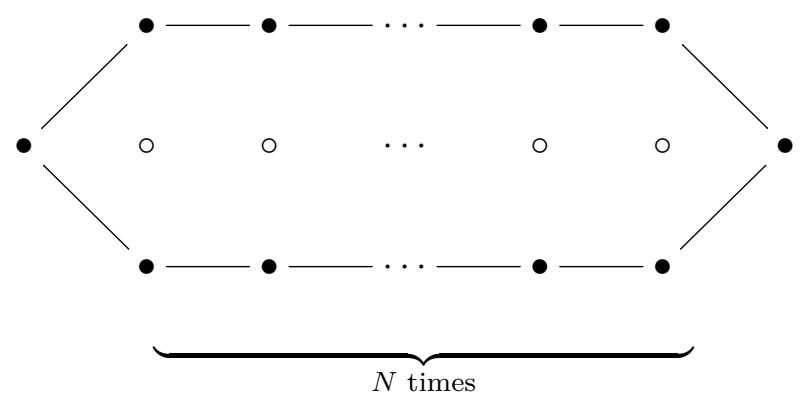

From the horizontal reduction it is manifest that we do not have any residual one-form symmetry: we obtain an $\mathrm{SU}(N+1)$ theory coupled to matter in the fundamental, which completely breaks the center symmetry, which is manifest from the following horizontal reduction/ruling

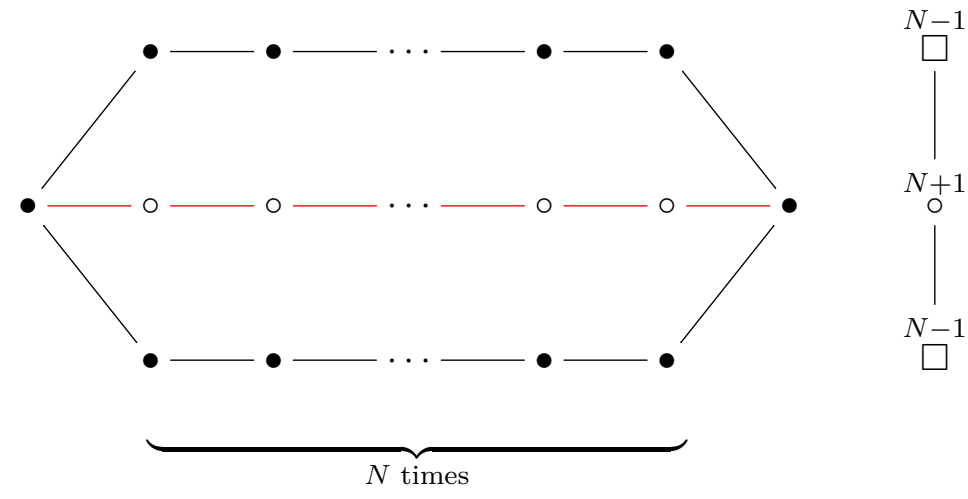


From the vertical reduction one obtains a quiver $\mathrm{SU}(2)$ gauge theory with bifundamental matter, therefore naively one could expect to preserve an overall diagonal $\mathbb{Z}_{2}$ action. Also in this case geometry reveals the presence of discrete theta terms: proceeding as above we can cut open this geometry in a way compatible with the ruling associated to the $\mathrm{SU}(2)$ quiver description

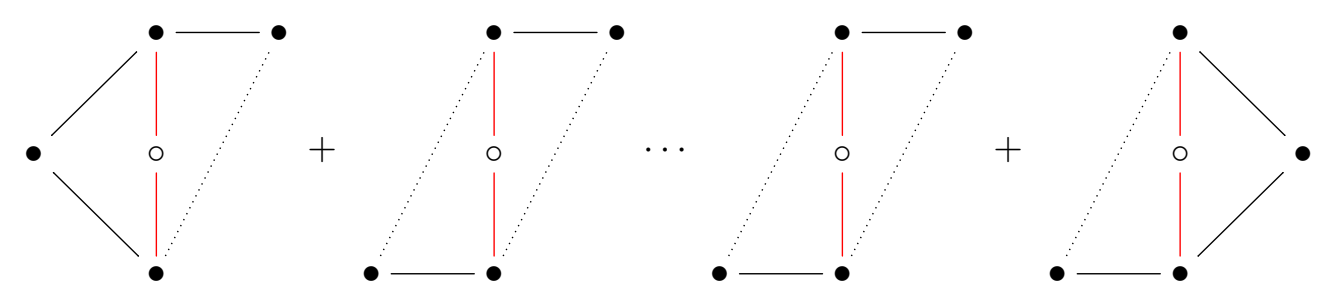

which corresponds to the surface-quiver diagram

$$
d P_{1}-\mathbb{F}_{0}-\cdots-\mathbb{F}_{0}-d P_{1}
$$

and the two $\mathrm{SU}(2)$ factors at the end of the quiver tails carry a nontrivial discrete theta term. For this reason the corresponding instantonic BPS states are charged with respect to the center symmetry thus breaking the overall diagonal $\mathbb{Z}_{2}$.

\subsection{Dualities and modified prescription for non-isolated toric singularities}

The example above corresponds to geometries that have curves of singularities giving rise to a non abelian $\mathrm{SU}(2 N-2)^{(0)}$ global symmetry. The corresponding singularity is nonisolated, and therefore the prescription we introduced in section 3.3 to compute the defect groups geometrically needs to be slightly modified. Motivated by the discussion above, it is natural to conjecture that whenever the geometry has non-compact curves of singularities corresponding to marked points on an outer edge of the toric diagram, the only triangles $T_{i}=\left\langle p_{i-1}, p_{i}, p_{i+1}\right\rangle$ that should be included in the computation are those where $p_{i}$ is not along an edge of the toric diagram. We will call the vertices appearing in such triangles "good" outer vertices. Let us consider the above example: the good outer vertices are in green

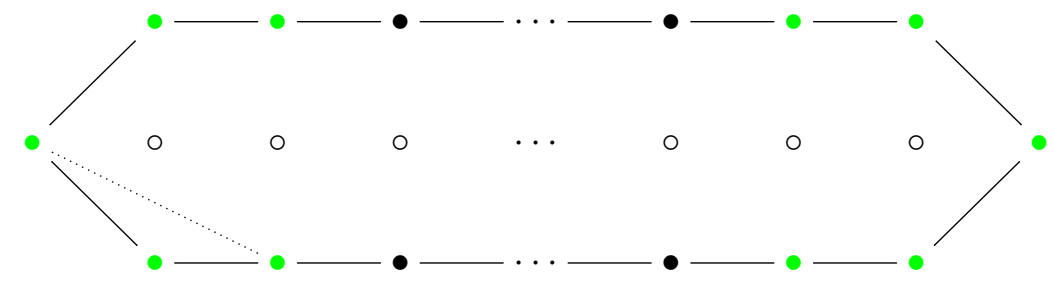

and it is clear that there is at least one outer triangle with minimal area (dashed line above), thus leading to a trivial defect group.

As a further consistency check let us consider the theory

$$
\underbrace{\mathrm{SU}(2)_{0} \times \cdots \times \mathrm{SU}(2)_{0}}_{N \text { times }}
$$



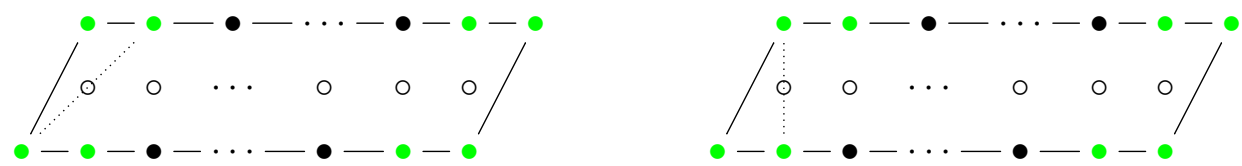

Figure 6. Computation of the defect group for the $\left(\mathrm{SU}(2)_{0}\right)^{N}$ theory: all the allowed triangles have twice the minimal area. Here we draw two of the four allowed triangles - the other two are the symmetric ones, on the other side of the figure.

is realized as a $\mathcal{T}_{\mathcal{V}_{6}}$ where $\mathcal{V}_{6}$ is the toric $\mathrm{CY}$ singularity with toric diagram

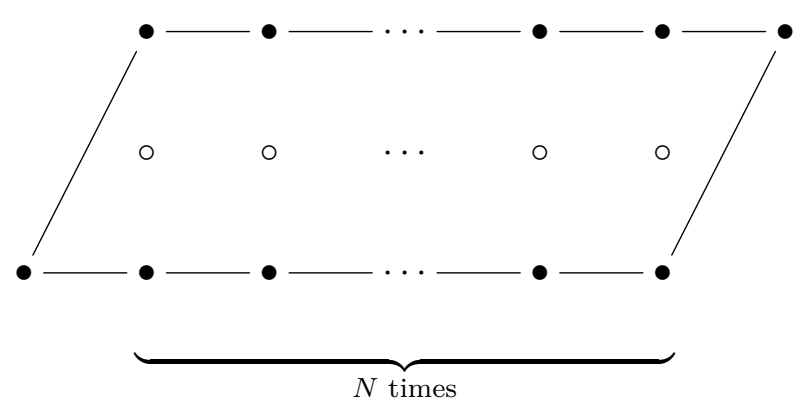

Clearly this does not admit a horizontal reduction, however the corresponding vertical reduction gives rise to the quiver theory in equation (5.13). According to the gauge theory analysis this geometry should correspond to a model that has a nontrivial $\mathbb{Z}_{2}^{(1)} \times \mathbb{Z}_{2}^{(2)}$ discrete higher symmetry group: the instantons are neutral with respect to the diagonal $\mathbb{Z}_{2}$ center simmetry, which is unbroken. The fact that all discrete theta terms are zero corresponds to the fact that this geometry has the surface-quiver diagram

$$
\mathbb{F}_{0}-\mathbb{F}_{0} \cdots-\mathbb{F}_{0}
$$

Coloring the good outer vertices according to our modified prescription we obtain the graph in figure 6: it is clear that the corresponding outer triangles obtained by connecting 3 green adjacent vertices all have area 1 . According to our prescription the corresponding defect group is $\mathbb{Z}_{2}^{(1)} \times \mathbb{Z}_{2}^{(2)}$, which clearly matches with the field theory prediction.

As a further consistency, let us consider another variation on the theme above, the theory

$$
\underbrace{\mathrm{SU}(K)_{0} \times \cdots \times \mathrm{SU}(K)_{0}}_{N \text { times }}
$$

is expected, from a similar token, to have a defect group $\mathbb{Z}_{K}^{(1)} \times \mathbb{Z}_{K}^{(2)}$, arising from the diagonal $\mathbb{Z}_{K}$ center symmetry which leaves the bifundamentals invariant. The corresponding colored 
toric diagram is a trapezium of height $K$ :

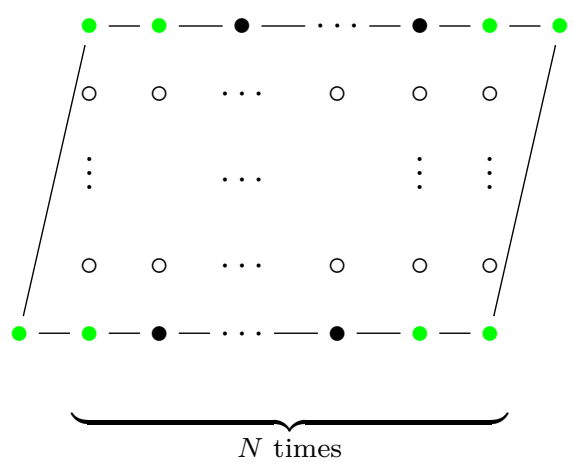

The corresponding allowed triangles all have area $K / 2$, and therefore the corresponding defect group is indeed $\mathbb{Z}_{K}^{(1)} \times \mathbb{Z}_{K}^{(2)}$ also from geometry, thus matching the gauge theory expectation.

Geometric interpretation. It is not very difficult to argue that the modified prescription that we have given is indeed the correct one geometrically. Recall that the key observation in the analysis in [100], reviewed in section 3.3, is that for the purposes of computing the torsion of $Y_{5}$ one can replace $Y_{5}$ by a chain of lens spaces $L_{n_{i}}$, connected on their non-trivial one-cycle. Each lens space is associated with a triangle $T_{i}$ formed by the three consecutive boundary vertices $\left\{p_{i-1}, p_{i}, p_{n+1}\right\}$, and $n_{i}$, the degree of the torsion group of the lens space, is twice the area of $T_{i}$.

Whenever we have a point $p_{i}$ along an edge, the triangle $T_{i}$ will have zero area, so this is formally $L_{0}$. If we define the lens space $L_{n}$ as a circle fibration over $S^{2}$ of degree $n$, then $L_{0} \cong S^{2} \times S^{1}$. This is the right answer from the toric geometry: whenever we have points along an edge, upon crepant resolution the local geometry of the $T^{2}$ fiber considered in [100] around the point is that of $S^{2} \times S^{1}$ - see figure 7. Connecting the $S^{1}$ factor to torsion cycles on either side is homotopically equivalent to connecting the torsion cycles directly, so effectively one can ignore the $L_{0}$ factors, which is the prescription we used above. Alternatively, we can include these triangles, and simply state that the prescription is still as in (3.25):

$$
\mathrm{Z}=\mathbb{Z}_{\operatorname{gcd}\left(n_{1}, \ldots, n_{v}\right)}
$$

taking into account that $\operatorname{gcd}(0, \ldots)=\operatorname{gcd}(\ldots)$.

In the next section, exploiting this prescription, we analyze several strongly coupled examples and determine the corresponding global structure. Having done that we give more applications in the context of other $5 \mathrm{~d}$ "dualities" involving strongly coupled conformal matter in section 6.4 .

\section{$6 \quad$ Non-Lagrangian examples}

In this section we exploit the formalism developed above to study the defect group and global structures of $5 \mathrm{~d}$ SCFTs without a $5 \mathrm{~d}$ gauge theory phase. 

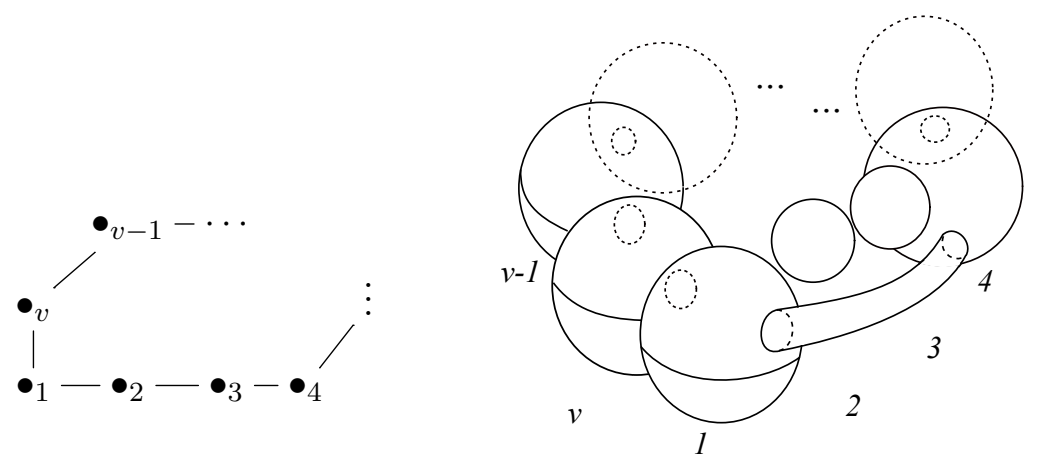

Figure 7. Schematic topology of $B_{3}^{L}$.

\subsection{The global structure of the $5 \mathrm{~d} E_{0}$ SCFT}

The simplest example of model that does not admit any gauge theory phase is given by the $5 \mathrm{~d} E_{0}$ SCFT associated to the toric canonical singularity $\mathbb{C}^{3} / \mathbb{Z}_{3}$, with toric diagram

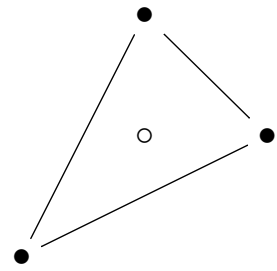

The naive defect group for the $E_{0}$ theory is ${ }^{12}$

$$
\mathbb{Z}_{3}^{(1)} \times \mathbb{Z}_{3}^{(2)}
$$

since in the toric diagram (6.1) there is a single outer triangle with area $3 / 2$. This can be understood both from the perspective of the charged defects and from the perspective of the corresponding charge operators.

The model has a non-trivial BPS string supported on the exceptional $\mathbb{P}^{2}$ which arises by resolving the singularity, as well as non-trivial BPS state corresponding to the only compact curve in the geometry $\mathcal{C}$. This BPS string has a nontrivial Dirac pairing with the corresponding BPS particles:

$$
\mathrm{D} \equiv \mathcal{C}_{x} \cdot \mathbf{E}=-3
$$

therefore the defect group for this model is

$$
\mathbb{D}=\left(\mathbb{Z}_{3}\right)_{M 2}^{(1)} \oplus\left(\mathbb{Z}_{3}^{(2)}\right)_{M 5}
$$

Let's now study this model from the perspective of the flux operators as well. The analysis of this model proceeds similarly to the Lagrangian cases we discussed in section 4 , but

\footnotetext{
${ }^{12}$ Here we are ignoring the $\mathbb{Z}_{3}^{(0)}$ symmetry coming from the isometries — we thank Kantaro Ohmori for pointing this out. In this particular case it is easy to see that it acts trivially on the fluxes, and therefore it is a global symmetry for all the theories in this class.
} 
now we have no gauge theoretical interpretation of the result. Nevertheless, if we had one we would expect the different global forms to be associated to possible choices of flux backgrounds in

$$
H^{2}\left(\mathcal{M}_{5} ; \mathrm{Z}\right)_{e} \times H^{3}\left(\mathcal{M}_{5} ; \mathrm{Z}\right)_{m},
$$

for some Z playing the role of the "center of the gauge group". We do obtain this structure from the M-theory construction: we now have

$$
H^{\bullet}\left(S^{5} / \mathbb{Z}_{3}\right)=\left\{\mathbb{Z}, 0, \mathbb{Z}_{3}, 0, \mathbb{Z}_{3}, \mathbb{Z}\right\}
$$

Using the Künneth formula as in section 3.2 we conclude that

$$
\begin{aligned}
& \text { Tor } H^{4}\left(\mathcal{M}_{5} \times\left(S^{5} / \mathbb{Z}_{3}\right)\right)=\left(H^{2}\left(\mathcal{M}_{5}\right) \otimes \text { Tor } H^{2}\left(S^{5} / \mathbb{Z}_{3}\right)\right) \oplus\left(H^{0}\left(\mathcal{M}_{5}\right) \otimes \text { Tor } H^{4}\left(S^{5} / \mathbb{Z}_{3}\right)\right) \\
& \text { Tor } H^{7}\left(\mathcal{M}_{5} \times\left(S^{5} / \mathbb{Z}_{3}\right)\right)=\left(H^{3}\left(\mathcal{M}_{5}\right) \otimes \text { Tor } H^{4}\left(S^{5} / \mathbb{Z}_{3}\right)\right) \oplus\left(H^{5}\left(\mathcal{M}_{5}\right) \otimes \text { Tor } H^{2}\left(S^{5} / \mathbb{Z}_{3}\right)\right)
\end{aligned}
$$

We see that we have room for $(-1)$-form and 4 -form background fluxes. We plan to investigate these in the future, for now we focus on the fluxes for the 1-form and the 2 -form higher symmetries for this model.

The charge operators for the higher 1- and 2- form symmetries of this theory are represented by the decomposition of the M-theory fluxes above. Notice that the M2 flux $\Phi_{a}$ is parametrized by a class $a \in H^{2}\left(\mathcal{M}_{5} ; \mathbb{Z}_{3}\right)$, while the M5 flux $\Psi_{b}$ is parametrized by a class $b \in H^{3}\left(\mathcal{M}_{5} ; \mathbb{Z}_{3}\right)$. We can decompose $a=\omega_{2} \otimes$ a where a $\in$ Tor $H^{2}\left(S^{5} / \mathbb{Z}_{3}\right)$ and $\omega_{2} \in H^{2}\left(\mathcal{M}_{5}\right)$, and $b=\omega_{3} \otimes \mathbf{b}$ where $\mathbf{b} \in$ Tor $H^{4}\left(S^{5} / \mathbb{Z}_{3}\right)$ and $\omega_{3} \in H^{3}\left(\mathcal{M}_{5}\right)$, then

$$
\Phi_{a} \Psi_{b}=e^{2 \pi i \mathrm{~L}(a, b)} \Psi_{b} \Phi_{a}
$$

where

$$
\mathrm{L}(a, b) \equiv \ell(\mathrm{a}, \mathrm{b})\left(\int_{\mathcal{M}_{5}} \omega_{2} \wedge \omega_{3}\right) .
$$

The second term in parenthesis gives the linking for the support of the two operators along $\mathcal{M}_{5}$, while the first term is given by the linking pairing

$$
\ell: H^{2}\left(S^{5} / \mathbb{Z}_{3}\right) \times H^{4}\left(S^{5} / \mathbb{Z}_{3}\right) \rightarrow \mathrm{U}(1)
$$

The only missing piece in order to show that this does indeed lead to a choice of global structure for this theory is showing that this linking pairing is nontrivial — but it necessarily is non-trivial due to being perfect. Indeed, the general computation in appendix B.2 gives

$$
\ell(1,1)=-\frac{1}{3}
$$

and therefore the possible choices of global structure for this model are the same as those of the $\mathfrak{s u}(3)$ theory in $4 \mathrm{~d}[77]$.

As pointed out in [102], this model is the 5d analogue of an Argyres-Douglas theory [103], and indeed it arises along the Coulomb branch of the $5 \mathrm{~d} \mathcal{N}=1$ gauge $\mathrm{SU}(2)_{\pi}$ 
gauge theory, precisely by suitable tuning, as manifest from the corresponding toric diagram - recall that $\mathrm{SU}(2)_{\pi}$ corresponds to the CY threefold obtained from a $d P_{1}$ base:

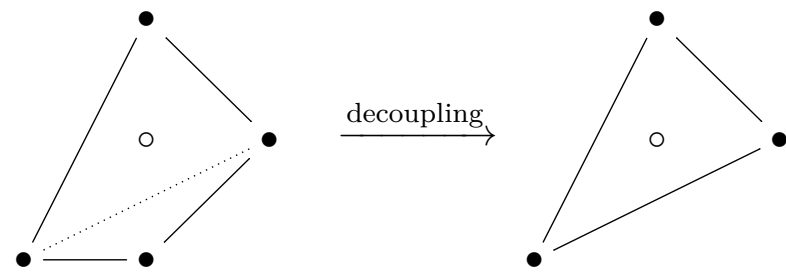

It is interesting to remark that, while $\mathrm{SU}(2)_{\pi}$ does not have nontrivial higher form symmetries, upon decoupling these symmetry emerge. Clearly the particles we are decoupling are charged with respect to this discrete symmetry and thus are breaking it explicitly in the $\mathrm{SU}(2)_{\pi}$ theory. The decoupling is achieved starting from an $\mathrm{SU}(2)_{\pi}$ gauge theory phase by a flop transition involving a massless BPS instanton. Naively one would expect a $\mathbb{Z}_{2}$ symmetry, however along this transition the symmetry enhances.

\subsection{The higher rank $5 \mathrm{~d} E_{0}$ theories}

The discussion in the previous section carries over word for word for the class of higher rank $5 \mathrm{~d} E_{0}$ Argyres-Douglas like theories, which correspond to the M-theory singularities $\mathbb{C}^{3} / \mathbb{Z}_{2 p+1}$. These models can be obtained by deforming $\mathrm{SU}(p)_{1}$ as follows [102]

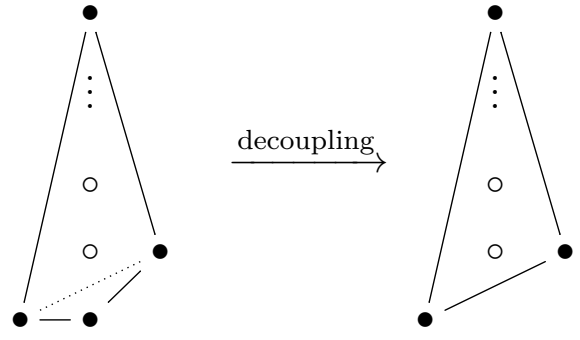

The gauge theory we begin with has trivial defect group, while the higher rank $E_{0}$ theory has defect group $^{13}$

$$
\mathbb{Z}_{2 p+1}^{(1)} \times \mathbb{Z}_{2 p+1}^{(2)} .
$$

An enhancement similar to the one observed in the previous section is in place. As we shall see below this enhancement is a feature for many descendants of the trinionic 5d SCFTs as well.

The global structure in this case is obtained by the same procedure. We have

$$
H^{\bullet}\left(S^{5} / \mathbb{Z}_{2 p+1}\right)=\left\{\mathbb{Z}, 0, \mathbb{Z}_{2 p+1}, 0, \mathbb{Z}_{2 p+1}, \mathbb{Z}\right\}
$$

and therefore, proceeding as in the previous section we obtain

$$
\begin{aligned}
& \text { Tor } H^{4}\left(\mathcal{M}_{5} \times\left(S^{5} / \mathbb{Z}_{3}\right)\right)=H^{2}\left(\mathcal{M}_{5} ; \mathbb{Z}_{2 p+1}\right) \oplus H^{0}\left(\mathcal{M}_{5} ; \mathbb{Z}_{2 p+1}\right) \\
& \text { Tor } H^{7}\left(\mathcal{M}_{5} \times\left(S^{5} / \mathbb{Z}_{3}\right)\right)=H^{3}\left(\mathcal{M}_{5} ; \mathbb{Z}_{2 p+1}\right) \oplus H^{5}\left(\mathcal{M}_{5} ; \mathbb{Z}_{2 p+1}\right) .
\end{aligned}
$$

\footnotetext{
${ }^{13}$ As in footnote 12 here we are ignoring the $\mathbb{Z}_{p}^{(0)}$ symmetry coming from the isometries. The same remark applies.
} 
Ignoring the (-1)-form and the 4-form symmetries, we focus on the M2 flux $\Phi_{a}$ parametrized by a class $a \in H^{2}\left(\mathcal{M}_{5} ; \mathbb{Z}_{2 p+1}\right)$, and on the M5 flux $\Psi_{b}$ parametrized by a class $b \in$ $H^{3}\left(\mathcal{M}_{5} ; \mathbb{Z}_{2 p+1}\right)$. We have that

$$
\Phi_{a} \Psi_{b}=e^{2 \pi i \mathrm{~L}(a, b)} \Psi_{b} \Phi_{a}
$$

and decomposing $a=\omega_{2} \otimes \mathrm{a}$ and $b=\omega_{3} \otimes \mathrm{b}$

$$
\mathrm{L}(a, b) \equiv \ell(\mathrm{a}, \mathrm{b})\left(\int_{\mathcal{M}_{5}} \omega_{2} \wedge \omega_{3}\right)
$$

where $\ell(a, b)$ is the linking pairing (see appendix B.2)

$$
\ell(1,1)=-\frac{1}{2 p+1},
$$

we see that this linking pairing has the same structure (up to a sign) as the pairing for the $4 \mathrm{~d} \mathfrak{s u}(2 p+1)$ algebras, and therefore the rank $p E_{0}$ theories have the same choices of global structures! These global structures have been worked out in [77], to which we refer the interested readers.

\subsection{The 5d trinions and their descendants}

Consider the singularity corresponding to the $5 \mathrm{~d} T_{N}$ theory [104]

$$
\mathcal{V}_{N} \equiv \frac{\mathbb{C}^{3}}{\mathbb{Z}_{N} \times \mathbb{Z}_{N}}
$$

The corresponding SCFT has 0-form global symmetry $\left(\mathrm{SU}(N)^{(0)}\right)^{3}$, which is manifest from the fact that there are three lines of singularities $\mathbb{C}^{2} / \mathbb{Z}_{N} \times \mathbb{C}$ meeting at the origin. The corresponding colored toric diagram is

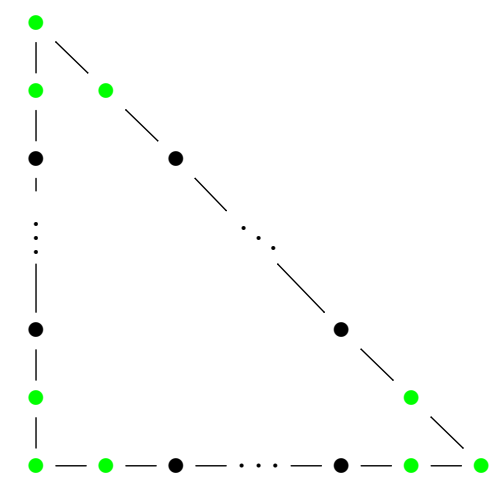

where each edge has $N+1$ bullets. It is manifest that there are triangles of minimal area above, therefore this theory has a trivial defect group. This fact can be understood easily because the $5 \mathrm{~d}$ trinion has a gauge theory phase $[22,23]$

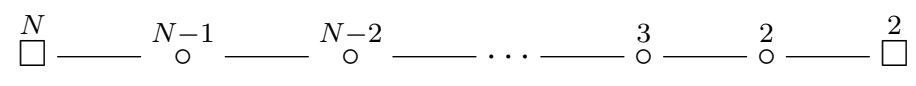

and the presence of matter in the fundamental is breaking the diagonal center symmetry. 


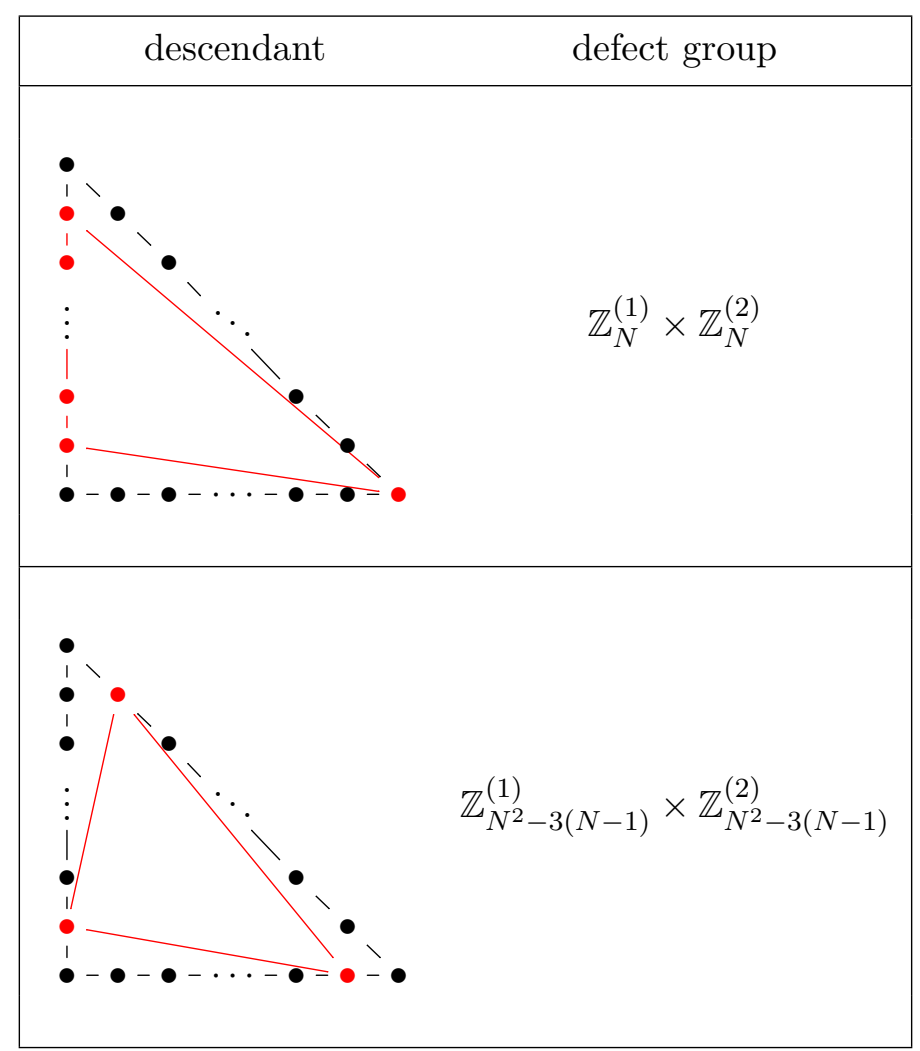

Figure 8. Examples of $\mathcal{T}_{N}$ descendants [105] with a nontrivial center symmetry. The one on the bottom is known as the $B_{N}$ theory [105].

The $\mathcal{T}_{N}$ theories have several descendant theories obtained by decoupling in [105]. As descendant theories are determined by decoupling, these correspond to those convex toric diagrams that embed in the ones associated to the $\mathcal{T}_{N}$ theories. In particular notice that all $\mathcal{T}_{K}$ theories with $K<N$ are always descendants of $\mathcal{T}_{N}$. Using our method is rather easy to identify graphically examples in this class that have non-trivial higher form symmetries by applying the following two criteria

1. Non-Lagrangian: the descendant geometries with a larger defect group typically do not admit a quiver gauge theory phase, which entails some of the horizontal, vertical and diagonal rulings must be obstructed;

2. High gcd: the outer green triangles should have areas with non-trivial greater common divisor

Some examples of descendant theories with a nontrivial higher form symmetry are listed in figure 8. A systematic analysis of the remaining cases is beyond the scope of the present note.

\subsection{More about 5d "dualities"}

It is interesting to remark that the $5 \mathrm{~d}$ quiver theory $\left(\mathrm{SU}(K)_{0}\right)^{N}$ has $5 \mathrm{~d}$ "duals" that are realized via quiver theories with strongly coupled trinionic $5 \mathrm{~d}$ conformal matter. As a 
concrete example, consider the theory $\left(\mathrm{SU}(4)_{0}\right)^{6}$, which belongs to the family of models we have analyzed in the previous paragraph. Considering the following ruling of this geometry:

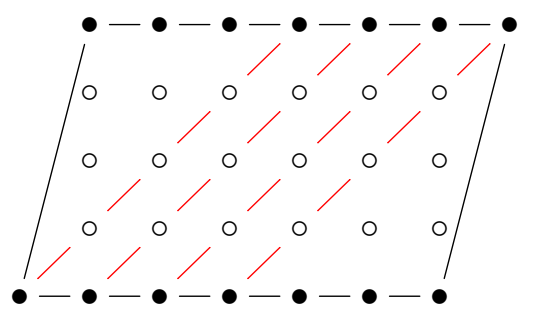

It is manifest that this model admits a different quiver description, namely

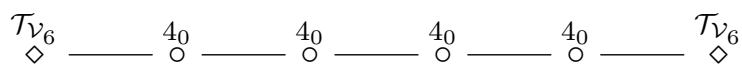

where, as usual, the edges denote bifundamentals, while nodes $\stackrel{N_{k}}{\circ}$ correspond to a gauge subsector $\mathrm{SU}(N)_{k}$, but we have introduced another kind of "meta-node," $\diamond$, which corresponds to a strongly coupled sector. Edges connecting the meta-nodes to a gauge node indicate the gauging of a zero-form global symmetry. In the context of this example, we have two meta-nodes associated to two copies of the same $\mathcal{T}_{\mathcal{V}_{6}}$ theory, where $\mathcal{V}_{6}$ is the toric canonical singularity associated to the toric diagram

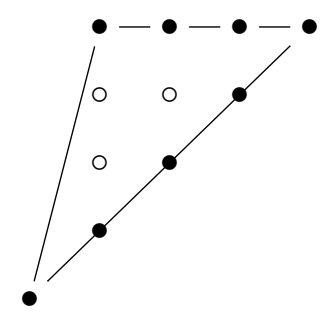

This model is among the descendant theories of the $5 \mathrm{~d} \mathcal{T}_{4}$ trinion and clearly has a global symmetry $\mathrm{SU}(4)^{(0)} \times \mathrm{SU}(3)^{(0)}$, and no 1-form symmetries. From the duality, it is manifest that the operators in this theory must be invariant under the $\mathbb{Z}_{4}$ center symmetry of SU(4), which survives the gauging, and provides the necessary structure for the gauged theory to have the $\mathbb{Z}_{4}$ center symmetry.

We can also consider gauging this flavor symmetry only, thus producing yet another 5d SCFT corresponding to the toric diagram below

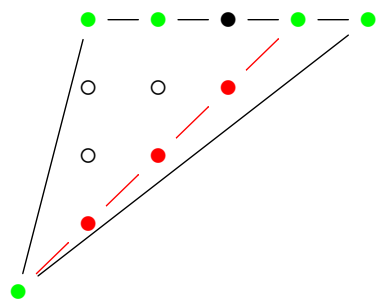

here we have marked in red the nodes corresponding to the SU(4) gauge symmetry, and in green the nodes which enters the computation of the defect groups. The resulting theory 
has quiver

$$
\stackrel{\mathcal{T}_{6}}{\diamond} \stackrel{4_{0}}{\circ}
$$

and defect group $\mathbb{Z}_{4}^{(1)} \times \mathbb{Z}_{4}^{(2)}$. Notice that we can introduce a nontrivial CS level for the $\mathrm{SU}(4)$ gauge theory, leading to the gauge theories

$$
\stackrel{\mathcal{T}_{\mathcal{V}_{6}}}{\diamond} \stackrel{4_{p}}{\stackrel{0}{\diamond}}
$$

with defect groups $\mathbb{Z}_{\mathrm{gcd}(4, p)}^{(1)} \times \mathbb{Z}_{\mathrm{gcd}(4, p)}^{(2)}$, corresponding to the following toric diagrams
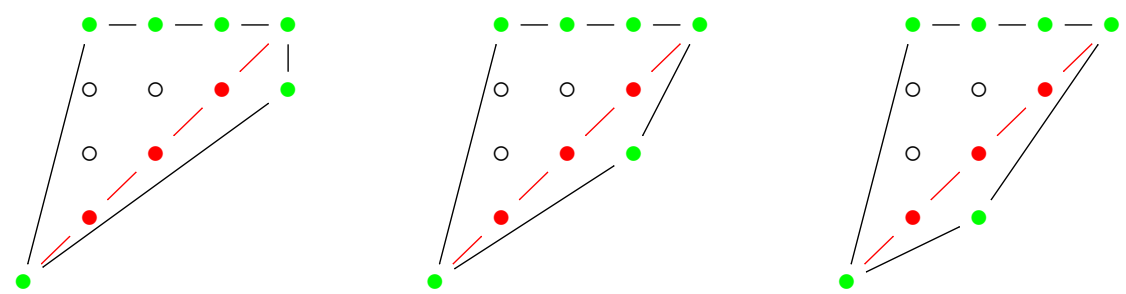

In these examples, the center of the gauge group is broken (partially, for $p=2$ ) by the charges of the corresponding instantons. It is interesting to remark that the second model has a different $5 \mathrm{~d}$ "dual" description

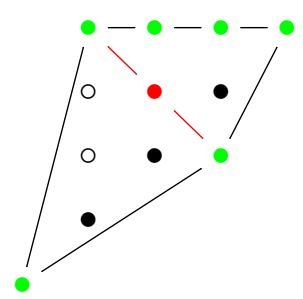

which has a generalized quiver

$$
\stackrel{\mathcal{T}_{1}}{\diamond} \stackrel{2_{0}}{\mathrm{O}} \longrightarrow \stackrel{\mathcal{T}_{2}}{\diamond}
$$

where we see another manifestation of the fact that the descendant of the $5 \mathrm{~d}$ trinions respect the center of the gauge group upon gauging, which in this case gives a defect group that is precisely $\mathbb{Z}_{2}^{(1)} \times \mathbb{Z}_{2}^{(2)}$.

These examples (which easily generalize) illustrate another application of 5 d "dualities" to understand the structure of the operators of strongly coupled 5d SCFTs. (A somewhat analogous argument, applied in reverse, was used in [69] to learn about the 1-form symmetries of the $T_{N}$ theories.)

\section{Global structure of $4 \mathrm{~d} \mathcal{N}=1$ theories and M-theory on $G_{2}$ spaces}

In this section we give an appetizer about the application of our method in the context of M-theory on spaces with $G_{2}$ holonomy. More precisely we consider here a geometric engineering on a space

$$
\mathcal{M}_{4} \times \mathcal{V}_{7}
$$


where $\mathcal{V}_{7}$ is a local $G_{2}$ space. For the applications we have in mind,

$$
\mathcal{V}_{7}=\mathcal{C}\left(Y_{6}\right)
$$

where $Y_{6}$ is a nearly Kähler manifold or an orbifold thereof - see e.g. [106]. It is wellknown that M-theory on a $G_{2}$ cone gives rise to four-dimensional quantum field theories with $\mathcal{N}=1$ supersymmetry (see e.g. [107] and references therein for a nice review). Our task in this section is to determine the global structure of one such theory.

Repeating the same arguments as above we expect this is determined from the structure of flux operators $\Phi_{M 2, a}$ with $a \in$ Tor $H^{4}\left(\mathcal{M}_{4} \times Y_{6}\right)$ and $\Psi_{M 5, b}$ with $b \in \operatorname{Tor} H^{7}\left(\mathcal{M}_{4} \times Y_{6}\right)$ (measuring torsional M2 and M5 charge, respectively). Again we are interested in studying the structure of

$$
\Phi_{M 2, a} \Psi_{M 5, b}=e^{2 \pi i \mathrm{~L}(a, b)} \Psi_{M 5, b} \Phi_{M 2, a}
$$

where

$$
\text { L: } \operatorname{Tor} H^{4}\left(\mathcal{M}_{4} \times Y_{6}\right) \times \operatorname{Tor} H^{7}\left(\mathcal{M}_{4} \times Y_{6}\right) \rightarrow \mathbb{Q} / \mathbb{Z}
$$

is the linking pairing in $\mathcal{M}_{4} \times Y_{6}$.

Assuming that $\mathcal{M}_{4}$ has no torsion we are interested in the cohomology groups

$$
\text { Tor } H^{m}\left(\mathcal{M}_{4} \times Y_{6}\right)=\sum_{i+j=m} H^{i}\left(\mathcal{M}_{4}\right) \otimes \operatorname{Tor} H^{j}\left(Y_{6}\right) \quad m=4,7
$$

Poincaré duality, together with the universal coefficient theorem, implies that

$$
\mathrm{Z}=\operatorname{Tor} H^{2}\left(Y_{6}\right)=\operatorname{Tor} H^{5}\left(Y_{6}\right) \quad \tilde{Z}=\text { Tor } H^{3}\left(Y_{6}\right)=\operatorname{Tor} H^{4}\left(Y_{6}\right)
$$

are the only nonzero torsional parts from the cohomology of $Y_{6}$, and therefore the relevant parts of $\mathbb{E}_{M}\left(\mathcal{M}_{4} \times Y_{6}\right)$ that can give rise to a nontrivial global structure are (by the universal coefficient theorem)

$$
\begin{aligned}
& \text { Tor } H^{4}\left(\mathcal{M}_{4} \times Y_{6}\right)=H^{2}\left(\mathcal{M}_{4}, \mathrm{Z}\right) \oplus H^{1}\left(\mathcal{M}_{4}, \tilde{Z}\right) \oplus H^{0}\left(\mathcal{M}_{4}, \tilde{Z}\right) \\
& \text { Tor } H^{7}\left(\mathcal{M}_{4} \times Y_{6}\right)=H^{2}\left(\mathcal{M}_{4}, \mathrm{Z}\right) \oplus H^{3}\left(\mathcal{M}_{4}, \tilde{Z}\right) \oplus H^{4}\left(\mathcal{M}_{4}, \tilde{Z}\right)
\end{aligned}
$$

where the direct summands above are paired along the vertical direction in the above equation, meaning that for this class of examples we have three distinct commuting Heisenberg algebras acting on $\mathcal{H}_{M}\left(\mathcal{M}_{4} \times Y_{6}\right)$.

From the above structure we see that from the first summand there are an electric and a magnetic $Z^{(1)}$ form symmetry, from the second summand we have similarly a $\tilde{Z}^{(0)}$ form and a $\tilde{Z}^{(2)}$ form symmetry, while the last summand corresponds to $\tilde{Z}^{(-1)}$ form and a $\tilde{Z}^{(3)}$ form symmetries. The defect group for this geometry is

$$
\mathbb{D}=\left(\mathbf{Z}_{M 2}^{(1)} \oplus \mathbf{Z}_{M 5}^{(1)}\right) \oplus\left(\tilde{\mathbf{Z}}_{M 2}^{(0)} \oplus \tilde{\mathbf{Z}}_{M 5}^{(2)}\right) \oplus\left(\tilde{\mathbf{Z}}_{M 2}^{(-1)} \oplus \tilde{\mathbf{Z}}_{M 5}^{(3)}\right)
$$

In this paper we are going to ignore the effects associated to (-1)-form symmetries and the 3-form symmetries. ${ }^{14}$

\footnotetext{
${ }^{14}$ These are somewhat more exotic and we defer their study to the future — see [88] for a reference about these.
} 
Each of the three summands in parenthesis in (7.7) belongs to a distinct Heisenberg factor, thus signalling a mixed 't Hooft anomaly for the defect group. The non-commuting 1-form symmetries in this case are the analogues of the electric and the magnetic 1-form symmetries in Yang-Mills theory in four-dimensions, so this hardly come as a surprise [108].

It is interesting to remark however that discrete 0 -form charge operators and 2-form charge operator do not commute. This entails that we cannot specify a background for the discrete 0 -form symmetries of one such model and a background flux for the 2-form symmetries simultaneously whenever $\mathcal{M}_{4}$ has non-trivial one-cycles and three-cycles. ${ }^{15}$ An interesting example of a manifold where this choice needs to be made is

$$
\mathcal{M}_{4}=S^{1} \times S^{3}
$$

We emphasize that the choice that we need to make here is independent of the choice that we make in the 1 -form symmetry sector (note that generically, $Z \neq \tilde{Z}$ ). So whenever our manifold $\mathcal{M}_{4}$ has non-trivial one and two-cycles, there are generically two independent choices of global structure to be made when computing partition functions. There are interesting partition functions of this sort, the simplest is

$$
\mathcal{M}_{4}=T^{2} \times S^{2} .
$$

\subsection{Example: $4 \mathrm{~d} \mathcal{N}=1 \mathrm{SYM}$}

Here we focus on a simple class of examples of this kind provided by the Bryant-Salamon $G_{2}$ metric on the spin bundle over $S^{3}$ (and some of its orbifolds) [109, 110]. This metric has topology $S^{3} \times \mathbb{R}^{4}$, and we can realize this space as a hyperbolic submanifold of $\mathbb{H} \times \mathbb{H} \simeq$ $\mathbb{C}^{2} \times \mathbb{C}^{2}$

$$
\left|q_{1}\right|^{2}-\left|q_{2}\right|^{2}=\left|z_{1,1}\right|^{2}+\left|z_{1,2}\right|^{2}-\left|z_{2,1}\right|^{2}-\left|z_{2,2}\right|^{2}=V \quad V \in \mathbb{R}_{>0}
$$

where $z_{i, j}$ are complex coordinates. From this presentation it is clear that we can orbifold this space with a discrete subgroup $\Gamma \subset \mathrm{SU}(2)$ acting only on one of the two sets of coordinates, thus obtaining a space that topologically is $S^{3} \times \mathbb{C}^{2} / \Gamma$. This space is known to geometrically engineer a four-dimensional $\mathcal{N}=1 \mathrm{SYM}$ theory in M-theory [111-113].

One feature of this $G_{2}$ space is that it is a cone $\mathcal{C}\left(S^{3} \times S^{3} / \Gamma\right)$. It is straightforward to compute

$$
H^{\bullet}\left(S^{3} \times S^{3} / \Gamma\right)=\left\{\mathbb{Z}, 0, \Gamma^{\mathrm{ab}}, \mathbb{Z} \oplus \mathbb{Z}, 0, \Gamma^{\mathrm{ab}}, \mathbb{Z}\right\}
$$

hence for this class of examples the two groups in (7.7) are given by

$$
\mathrm{Z}=\Gamma^{\mathrm{ab}}=Z\left(G_{\Gamma}\right) \quad \tilde{\mathrm{Z}}=0
$$

therefore in this specific model there only one Heisenberg algebra. More precisely, for fluxes labelled by $a_{i}=(\omega \otimes \ell)_{i} \in H^{2}\left(\mathcal{M}_{4}\right) \otimes \mathrm{Z}$ we have

$$
\Phi_{M 2, a_{1}} \Psi_{M 5, a_{2}}=\exp \left(2 \pi i \mathrm{~L}_{\Gamma}\left(\ell_{1}, \ell_{2}\right) \int_{\mathcal{M}_{4}} \omega_{1} \wedge \omega_{2}\right) \Psi_{M 5, a_{2}} \Phi_{M 2, a_{1}}
$$

\footnotetext{
${ }^{15}$ Ultimately this effect is related to the fact that scalars are dual to tensors in four dimensions.
} 
where $L_{\Gamma}$ is the same pairing we introduced in section 2 . We thus reproduce the statement about the mixed 't Hooft anomaly among the $Z\left(G_{\Gamma}\right)_{M 2}^{(1)}$ and the $Z\left(G_{\Gamma}\right)_{M 5}^{(1)}$ symmetries in the defect group mentioned in the introduction of this paper.

More examples of $G_{2}$ cones are available in the literature. The global structures of these models are interesting and can be analyzed using our method. We will address this question in future work.

\section{Acknowledgments}

We thank Bobby Acharya, Francesco Benini, Cyril Closset, Stefano Cremonesi, Lorenzo Foscolo, Fernando Marchesano and Maxim Zabzine for discussions. This project has received funding from the European Research Council (ERC) under the European Union's Horizon 2020 research and innovation programme (grant agreement No. 851931). I.G.-E. is supported in part by STFC consolidated grant ST/P000371/1. This project was also supported in part by STFC grant with project reference ST/T506035/1.

\section{A The Mori cone for $\mathcal{C}_{\mathbb{R}}\left(Y^{p, q}\right)$}

In this section we will study in detail the structure of the Mori cone for the Calabi-Yau cone over $Y^{p, q}$ [101], which we denote by $\mathcal{C}_{\mathbb{R}}\left(Y^{p, q}\right)$. This Calabi-Yau threefold is toric, which simplifies the analysis of the relevant geometry. We refer the reader to [94] for general background on toric geometry and [95-97] for introductions aimed to physicists. The computer algebra program SAGE contains very useful implementations of the toric algorithms that we use [99].

Define $l:=p-q$. We can take the points in the toric diagram for $\mathcal{C}_{\mathbb{R}}\left(Y^{p, q}\right)$ to be $P_{1}=(-1,1), P_{3}=(l, 0)$ and $I_{i}=(0, i), i \in\{0,1, \cdots, p\}$. We choose the triangulation as in figure 4 , that is, such that the 3 -dimensional cones are of the form $\left(P_{1}, I_{k}, I_{k+1}\right)$ and $\left(P_{3}, I_{k}, I_{k+1}\right)$ with $k \in\{0,1,2, \cdots, p-1\}$.

We can construct a (non-minimal) basis of generating curves by taking intersections of toric divisors. The intersection numbers of the compact curves constructed in this way and the toric divisors are given in table 2. The Mori cone is spanned by compact curves corresponding to 2-dimensional cones. Thus, the number of the generators of the Mori cone equals to the number of independent 2-cycles. From our discussion in (3.18) we find that the number of independent compact 2-cycles is $p$, so this is the dimension of the Mori cone. We denote the Mori cone generators $C_{1}, \ldots, C_{p}$. Any two curves are linearly equivalent iff their intersection with all toric divisors are the same, so the problem of determining the $C_{i}$ reduces to finding a basis of linearly independent rows in table 2. From the table we can deduce the equivalence relations

$$
P_{1} \cdot I_{k} \equiv P_{3} \cdot I_{k}, \quad I_{k-1} \cdot I_{k}-I_{k} \cdot I_{k+1} \equiv(l-2 k) P_{1} \cdot I_{k},
$$

where $k \in\{1,2, \cdots,(p-1)\}$. Thus, we may choose the Mori cone generators to be

$$
C_{k}=P_{1} \cdot I_{k}, \quad C_{p}=I_{0} \cdot I_{1} .
$$




\begin{tabular}{|l|c|c|c|c|c|c|c|c|c|c|c|c|}
\hline Curve & $P_{1}$ & $P_{3}$ & $I_{0}$ & $I_{1}$ & $I_{2}$ & $\cdots$ & $I_{k-1}$ & $I_{k}$ & $I_{k+1}$ & $\cdots$ & $I_{p-1}$ & $I_{p}$ \\
\hline$P_{1} \cdot I_{1}$ & 0 & 0 & 1 & -2 & 1 & $\cdots$ & 0 & 0 & 0 & $\cdots$ & 0 & 0 \\
\hline$\cdots$ & & & & & & & & & & & & \\
\hline$P_{1} \cdot I_{k}$ & 0 & 0 & 0 & 0 & 0 & $\cdots$ & 1 & -2 & 1 & $\cdots$ & 0 & 0 \\
\hline$\cdots$ & & & & & & & & & & & & \\
\hline$I_{0} \cdot I_{1}$ & 1 & 1 & $l-2$ & $-l$ & 0 & $\cdots$ & 0 & 0 & 0 & $\cdots$ & 0 & 0 \\
\hline$I_{k} \cdot I_{k+1}$ & 1 & 1 & 0 & 0 & 0 & $\cdots$ & 0 & $l-2 k-2$ & $-l+2 k$ & $\cdots$ & 0 & 0 \\
\hline$\cdots$ & & & & & & & & & & & & \\
\hline$I_{p-1} \cdot I_{p}$ & 1 & 1 & 0 & 0 & $\cdots$ & 0 & 0 & 0 & 0 & $\cdots$ & $l-2 p$ & $-l+2(p-1)$ \\
\hline
\end{tabular}

Table 2. The intersection numbers of the $(2 P-1)$ compact curves $P_{1} \cdot I_{i}, I_{i} \cdot I_{i-1}$ and the $(P+3)$ points $P_{1}=(-1,1), P_{3}=(l, 0)$ and $I_{i}=(0, i)$, where $i \in\{0,1, \cdots, p\}$, and $k \in\{1,2, \cdots,(p-1)\}$. We have omitted the result for the curves $P_{3} \cdot I_{k}$ as they give the same intersection numbers as $P_{1} \cdot I_{k}$ for each fixed $k$.

\section{B Linking forms}

\section{B.1 $\mathcal{C}_{\mathbb{R}}\left(Y^{p, q}\right)$}

The intersection form $Q_{4}\left(Q_{2}=Q_{4}^{T}\right)$ between 4-cycles (2-cycles) and 2-cycles (4-cycles) can be easily read from the Mori cone generators to be

$$
Q_{4}=\left(\begin{array}{ccccccccc}
-2 & 1 & 0 & 0 & \cdots & 0 & 0 & 0 & 0 \\
1 & -2 & 1 & 0 & \cdots & 0 & 0 & 0 & 0 \\
\vdots & & & & & & & & \\
0 & 0 & 0 & 0 & \cdots & 0 & 1 & -2 & 1 \\
0 & 0 & 0 & 0 & \cdots & 0 & 0 & 1 & -2 \\
-l & 0 & 0 & 0 & \cdots & 0 & 0 & 0 & 0
\end{array}\right),
$$

for even $l$, where -2 in the last row is in the column $l / 2$ of $Q_{4}$ or in a more compact notation

$$
Q_{4}=\left(q_{i, j}\right), \quad q_{i, j}= \begin{cases}\delta_{i, j-1}-2 \delta_{i, j}+\delta_{i, j+1}, & \text { for } i, j \in\{1,2, \cdots, p-1\} \\ -l & \text { for } i=p \text { and } j=1 \\ 0, & \text { otherwise }\end{cases}
$$

Now, we can calculate the homology groups of $Y^{p, q}$ using (3.11). We can easily determine the kernel and the cokernel of $Q_{4}$ by finding the Smith normal form of $Q_{4}$, which we call $S_{4}$. We find that $S_{4}$ has the form

$$
S_{4}=\left(\begin{array}{ccccccc}
1 & 0 & 0 & \cdots & 0 & 0 & 0 \\
0 & 1 & 0 & \cdots & 0 & 0 & 0 \\
\vdots & & & & & & \\
0 & 0 & 0 & \cdots & 0 & 1 & 0 \\
0 & 0 & 0 & \cdots & 0 & 0 & \operatorname{gcd}(p, q) \\
0 & 0 & 0 & \cdots & 0 & 0 & 0
\end{array}\right)
$$


or in more compact notation

$$
Q_{4}=\left(s_{i, j}\right), \quad s_{i, j}= \begin{cases}\delta_{i, j}, & \text { for } i, j \in\{1,2, \cdots, p-1\}, \\ \operatorname{gcd}(p, q) \delta_{i, p-1} \delta_{j, p-1}, & \text { for } i=j=p-1, \\ 0, & \text { otherwise. }\end{cases}
$$

Hence, the image of $Q_{4}$ is $\operatorname{Im}\left(Q_{4}\right)=\mathbb{Z}^{p-2}+\operatorname{gcd}(p, q) \mathbb{Z}$ and its kernel is zero. We have

$$
\begin{aligned}
& H_{3}\left(Y^{p, q}\right)=\operatorname{coker}\left(Q_{4}\right)=\mathbb{Z}+\mathbb{Z}_{\operatorname{gcd}(p, q)}, \\
& H_{4}\left(Y^{p, q}\right)=\operatorname{ker}\left(Q_{4}\right)=0 .
\end{aligned}
$$

Similarly, since $Q_{2}=Q_{4}^{T}$ we find

$$
H_{2}\left(Y^{p, q}\right)=\operatorname{ker}\left(Q_{2}\right)=\mathbb{Z}, \quad H_{1}\left(Y^{p, q}\right)=\operatorname{coker}\left(Q_{2}\right)=\mathbb{Z}_{\operatorname{gcd}(p, q)} .
$$

We now want to compute the linking pairing

$$
\mathrm{L}_{Y^{p, q}}: \text { Tor } H_{p-1}\left(Y^{p, q}\right) \times \text { Tor } H_{n-p-1}\left(Y^{p, q}\right) \rightarrow \mathbb{Q} / \mathbb{Z} .
$$

In our case the only homology groups with non-trivial torsion are $H_{3}\left(Y^{p, q}\right)$ and $H_{1}\left(Y^{p, q}\right)$ so we may compute the linking of 3-cycles and 1-cycles as follows. From (3.17)

$$
L\left(\partial \alpha_{i}^{\prime *}, \bar{\partial} \beta_{j}^{\prime *}\right)=q^{-1}\left(\alpha_{i}^{\prime *}, \beta_{j}^{* *}\right)=q_{i j}^{-1}(\bmod 1) .
$$

We find

$$
q_{i, k}^{-1}= \begin{cases}(i-j)+(p-j) c / 2, & \text { for } i \geq j, j<p \text { and } i<p-1 \\ (p-j) c / 2, & \text { for } i<j, j<p \text { and } i<p-1 \\ (p-2-j) / 2+(p-j) c / 2, & \text { for } j<p \text { and } i=p-1 \\ -i / l-p c /(2 l), & \text { for } j=p \text { and } i<p-1 \\ (1 / l-p /(2 l))-p c /(2 l), & \text { for } j=p \text { and } i=p-1,\end{cases}
$$

such that $q^{-1} q=I$. All that remains is to find the generators $\alpha_{i}^{\prime *}$ and $\beta_{j}^{\prime *}$ defined above. This may be done by tracking how the generators in the basis defined by the matrix $q^{(T)}$ change as we switch basis by writing the matrix in its Smith normal form $S^{(T)}$. Given the form of our matrix $q$ in (B.1)), for $\beta_{i}^{*}$ and $\beta_{j}^{\prime *}$ the generators of $\operatorname{Hom}\left(H_{4}\left(X_{6}\right), \mathbb{Z}\right)$ in the $q$ basis and the $S$ basis, respectively, we find that, $\beta_{p-1}^{* *}=\beta_{p-1}^{*}$ where $\partial \beta_{p-1}^{*}$ is the generator of Tor $H_{1}(Y)$. Similarly, for $\alpha_{\bar{i}}^{*}$ and $\alpha_{\bar{j}}^{\prime *}$ the generators of $\operatorname{Hom}\left(H_{2}(X), \mathbb{Z}\right)$ in the $q^{T}$ basis, and the $S^{T}$ basis, respectively, we find $\alpha_{p}^{*}=p^{\prime} \alpha_{1}^{*}+q^{\prime} \alpha_{p}^{*}$ such that, $\partial \alpha_{p}^{*}$ is the generator of Tor $H_{3}\left(Y^{p, q}\right)$ where, $p^{\prime}=\frac{p}{\operatorname{gcd}(p, q)}$ and $q^{\prime}=\frac{l p-l}{\operatorname{gcd}(p, q)}$. Therefore, the linking number is

$$
\begin{aligned}
L\left(\partial \alpha_{p}{ }^{*}, \bar{\partial} \beta_{p-1}^{*}\right) & =L\left(p^{\prime} \partial \alpha_{1}^{*}+q^{\prime} \partial \alpha_{p}^{*}, \bar{\partial} \beta_{p-1}^{*}\right) \\
& =p^{\prime} q_{1, p-1}^{-1}+q^{\prime} q_{p, p-1}^{-1} \\
& =-\frac{1}{\operatorname{gcd}(p, q)} \quad(\bmod 1)
\end{aligned}
$$

using (B.6) and the bilinearity of the linking pairing. 


\begin{tabular}{|l|c|c|c|c|c|c|c|c|c|c|c|c|}
\hline Curve & $a$ & $b$ & $I_{0}$ & $I_{1}$ & $I_{2}$ & $\cdots$ & $I_{k-1}$ & $I_{k}$ & $I_{k+1}$ & $\cdots$ & $I_{n-1}$ & $I_{n}$ \\
\hline$a \cdot I_{1}$ & 0 & 0 & 1 & -2 & 1 & $\cdots$ & 0 & 0 & 0 & $\cdots$ & 0 & 0 \\
\hline$\cdots$ & & & & & & & & & & & & \\
\hline$a \cdot I_{k}$ & 0 & 0 & 0 & 0 & 0 & $\cdots$ & 1 & -2 & 1 & $\cdots$ & 0 & 0 \\
\hline$\cdots$ & & & & & & & & & & & & \\
\hline$I_{0} \cdot I_{1}$ & 1 & 1 & -3 & 1 & 0 & $\cdots$ & 0 & 0 & 0 & $\cdots$ & 0 & 0 \\
\hline$I_{k} \cdot I_{k+1}$ & 1 & 1 & 0 & 0 & 0 & $\cdots$ & 0 & $-3-2 k$ & $2 k+1$ & $\cdots$ & 0 & 0 \\
\hline$\cdots$ & & & & & & & & & & & & \\
\hline$I_{n-1} \cdot I_{n}$ & 1 & 1 & 0 & 0 & $\cdots$ & 0 & 0 & 0 & 0 & $\cdots$ & $-2 n-1$ & $2 n-1$ \\
\hline
\end{tabular}

Table 3. The intersection numbers of the $(2 P-1)$ compact curves $a \cdot I_{k}, I_{k} \cdot I_{k-1}$ and the $(n+3)$ points $a=(-1,0), b=(1,-1)$ and $I_{i}=(0, i)$, where $i \in\{0,1,2, \cdots, n\}$ and $k \in\{0,1,2, \cdots,(n-1)\}$. We have omitted the result for the curves $b \cdot I_{k}$ and $a \cdot I_{0}$ as they give the same intersection numbers as $a \cdot I_{k}$ and $I_{0} \cdot I_{1}$ for each fixed $k$, respectively.

\section{B.2 $\mathbb{C}^{3} / \mathbb{Z}_{2 n+1}$}

Let $a=(-1,0), b=(1,-1)$ and $I_{i}=(0, i)$ be the points on the toric diagram with $i=0,1,2, \cdots, n$, and choose the triangulation such that the 3 -dimensional cones are of the form $\left(a, b, I_{0}\right),\left(a, I_{k}, I_{k+1}\right)$ and $\left(b, I_{k}, I_{k+1}\right)$, where $k=0,1,2, \cdots, n-1$. As before, from the toric diagram we have

$$
H_{2}\left(X_{6}\right)=H_{4}\left(X_{6}\right)=\mathbb{Z}^{n} .
$$

From the intersection numbers given in table 3 , we deduce the equivalence relations (by subtracting the two relevant rows in terms of $k$ for the latter relation)

$$
a \cdot I_{i} \equiv b \cdot I_{i}, \quad a \cdot I_{0} \equiv I_{0} \cdot I_{1}, \quad I_{k+1} \cdot I_{k+2}-I_{k} \cdot I_{k+1} \equiv(3+2 k) a \cdot I_{k} .
$$

Therefore, we can choose the Mori cone generators $C_{k}$ to be the rows of table given by the intersection numbers for $a \cdot I_{k}$

$$
C_{k}=a \cdot I_{k}
$$

The intersection form is

$$
q_{i, j}= \begin{cases}\delta_{i, j}-2 \delta_{i, j-1}+\delta_{i, j-2}, & \text { for } \quad i \in\{1,2, . . n-1\}, j \in\{1,2, . . n\} \\ -3 \delta_{1, j}+\delta_{2, j}, & \text { for } i=n, j \in\{1,2, . . n\}\end{cases}
$$

which has Smith normal form

$$
S_{i, j}= \begin{cases}\delta_{i, j}, & \text { for } \quad i, j \in\{1,2, . . n-1\} \\ 2 n+1, & \text { for } \quad i, j=n\end{cases}
$$

From this we find

$$
\begin{aligned}
& H_{1}\left(Y_{5}\right)=H_{3}\left(Y_{5}\right)=\operatorname{coker}(Q)=\mathbb{Z}_{2 n+1}, \\
& H_{2}\left(Y_{5}\right)=H_{4}\left(Y_{5}\right)=\operatorname{ker}(Q)=0 .
\end{aligned}
$$


Now, to find the linking number, we track the effect on the generators as we write $Q$ in its Smith normal form. We find $\alpha_{n}^{*}$ and $\beta_{n}^{*}$ to be the generators of $\operatorname{Hom}\left(H_{2}(X), \mathbb{Z}\right)$ and $\operatorname{Hom}\left(H_{4}\left(X_{6}\right), \mathbb{Z}\right)$, respectively such that, $\partial \alpha_{n}^{*}$ and $\partial \beta_{n}^{*}$ are the generators of Tor $H_{3}\left(Y_{5}\right)$ and Tor $H_{1}\left(Y_{5}\right)$, respectively. It can be shown that the inverse of $q_{i, j}$ is

$$
-(2 n+1) q_{i, j}^{-1}= \begin{cases}(2 i-1)(n-j), & \text { for } j<n \text { and } j+1 \geq i \\ (n-i+1)(2 j+1), & \text { for } j+1 \leq i \\ (n-i+1), & \text { for } j=n\end{cases}
$$

i.e. $q_{n n}^{-1}=-\frac{1}{2 n+1}$, and so we have

$$
L\left(\partial \alpha_{n}^{*}, \partial \beta_{n}^{*}\right)=q_{n n}^{-1}=-\frac{1}{2 n+1} \bmod 1 .
$$

Open Access. This article is distributed under the terms of the Creative Commons Attribution License (CC-BY 4.0), which permits any use, distribution and reproduction in any medium, provided the original author(s) and source are credited.

\section{References}

[1] M. Del Zotto, J.J. Heckman, D.S. Park and T. Rudelius, On the Defect Group of a $6 D$ SCFT, Lett. Math. Phys. 106 (2016) 765 [arXiv:1503. 04806] [INSPIRE].

[2] A. Kapustin and N. Seiberg, Coupling a QFT to a TQFT and Duality, JHEP 04 (2014) 001 [arXiv: 1401.0740] [INSPIRE].

[3] D. Gaiotto, A. Kapustin, N. Seiberg and B. Willett, Generalized Global Symmetries, JHEP 02 (2015) 172 [arXiv: 1412.5148] [INSPIRE].

[4] E. Sharpe, Notes on generalized global symmetries in QFT, Fortsch. Phys. 63 (2015) 659 [arXiv: 1508.04770] [INSPIRE].

[5] C. Córdova, T.T. Dumitrescu and K. Intriligator, Exploring 2-Group Global Symmetries, JHEP 02 (2019) 184 [arXiv:1802.04790] [INSPIRE].

[6] D.S. Freed, G.W. Moore and G. Segal, The Uncertainty of Fluxes, Commun. Math. Phys. 271 (2007) 247 [hep-th/0605198] [inSPIRE].

[7] D.S. Freed, G.W. Moore and G. Segal, Heisenberg Groups and Noncommutative Fluxes, Annals Phys. 322 (2007) 236 [hep-th/0605200] [INSPIRE].

[8] I. García Etxebarria, B. Heidenreich and D. Regalado, IIB flux non-commutativity and the global structure of field theories, JHEP 10 (2019) 169 [arXiv:1908.08027] [INSPIRE].

[9] E. Witten, Geometric Langlands From Six Dimensions, arXiv:0905.2720 [InSPIRE].

[10] J.J. Heckman and L. Tizzano, 6D Fractional Quantum Hall Effect, JHEP 05 (2018) 120 [arXiv: 1708.02250] [INSPIRE].

[11] S. Gukov, D. Pei, P. Putrov and C. Vafa, 4-manifolds and topological modular forms, arXiv: 1811.07884 [INSPIRE].

[12] J. Eckhard, H. Kim, S. Schäfer-Nameki and B. Willett, Higher-Form Symmetries, Bethe Vacua, and the 3d-3d Correspondence, JHEP 01 (2020) 101 [arXiv:1910.14086] [InSPIRE]. 
[13] A. Dabholkar, P. Putrov and E. Witten, Duality and Mock Modularity, SciPost Phys. 9 (2020) 072 [arXiv: 2004.14387] [INSPIRE].

[14] C. Cordova, T.T. Dumitrescu and K. Intriligator, Multiplets of Superconformal Symmetry in Diverse Dimensions, JHEP 03 (2019) 163 [arXiv:1612.00809] [INSPIRE].

[15] C.-M. Chang, 5d and 6d SCFTs Have No Weak Coupling Limit, JHEP 09 (2019) 016 [arXiv: 1810.04169] [INSPIRE].

[16] N. Seiberg, Five-dimensional SUSY field theories, nontrivial fixed points and string dynamics, Phys. Lett. B 388 (1996) 753 [hep-th/9608111] [InSPIRE].

[17] D.R. Morrison and N. Seiberg, Extremal transitions and five-dimensional supersymmetric field theories, Nucl. Phys. B 483 (1997) 229 [hep-th/9609070] [InSPIRE].

[18] K.A. Intriligator, D.R. Morrison and N. Seiberg, Five-dimensional supersymmetric gauge theories and degenerations of Calabi-Yau spaces, Nucl. Phys. B 497 (1997) 56 [hep-th/9702198] [INSPIRE].

[19] O. Aharony and A. Hanany, Branes, superpotentials and superconformal fixed points, Nucl. Phys. B 504 (1997) 239 [hep-th/9704170] [InSPIRE].

[20] N.C. Leung and C. Vafa, Branes and toric geometry, Adv. Theor. Math. Phys. 2 (1998) 91 [hep-th/9711013] [INSPIRE].

[21] O. Bergman, D. Rodríguez-Gómez and G. Zafrir, Discrete $\theta$ and the $5 d$ superconformal index, JHEP 01 (2014) 079 [arXiv:1310.2150] [INSPIRE].

[22] H. Hayashi, Y. Tachikawa and K. Yonekura, Mass-deformed $T_{N}$ as a linear quiver, JHEP 02 (2015) 089 [arXiv: 1410.6868] [INSPIRE].

[23] O. Bergman and G. Zafrir, Lifting 4d dualities to 5d, JHEP 04 (2015) 141 [arXiv: 1410.2806] [INSPIRE].

[24] G. Zafrir, Duality and enhancement of symmetry in 5d gauge theories, JHEP 12 (2014) 116 [arXiv: 1408.4040] [INSPIRE].

[25] M. Del Zotto, J.J. Heckman, A. Tomasiello and C. Vafa, 6d Conformal Matter, JHEP 02 (2015) 054 [arXiv: 1407.6359] [inSPIRE].

[26] O. Bergman and G. Zafrir, 5d fixed points from brane webs and O7-planes, JHEP 12 (2015) 163 [arXiv: 1507.03860] [INSPIRE].

[27] G. Zafrir, Brane webs and O5-planes, JHEP 03 (2016) 109 [arXiv:1512.08114] [InSPIRE].

[28] H.-C. Kim, Line defects and 5d instanton partition functions, JHEP 03 (2016) 199 [arXiv:1601.06841] [INSPIRE].

[29] M. Del Zotto, C. Vafa and D. Xie, Geometric engineering, mirror symmetry and $6 \mathrm{~d}_{(1,0)} \rightarrow 4 \mathrm{~d}_{(\mathcal{N}=2)}$, JHEP $11(2015) 123$ [arXiv: 1504.08348] [INSPIRE].

[30] H. Hayashi, S.-S. Kim, K. Lee, M. Taki and F. Yagi, A new $5 d$ description of $6 d$ D-type minimal conformal matter, JHEP 08 (2015) 097 [arXiv:1505.04439] [INSPIRE].

[31] H. Hayashi, S.-S. Kim, K. Lee, M. Taki and F. Yagi, More on $5 d$ descriptions of $6 d$ SCFTs, JHEP 10 (2016) 126 [arXiv: 1512.08239] [INSPIRE].

[32] H. Hayashi, S.-S. Kim, K. Lee and F. Yagi, Equivalence of several descriptions for $6 d$ SCFT, JHEP 01 (2017) 093 [arXiv:1607.07786] [INSPIRE].

[33] D. Xie and S.-T. Yau, Three dimensional canonical singularity and five dimensional $\mathcal{N}=1$ SCFT, JHEP 06 (2017) 134 [arXiv:1704.00799] [INSPIRE]. 
[34] M. Del Zotto, J.J. Heckman and D.R. Morrison, 6D SCFTs and Phases of $5 D$ Theories, JHEP 09 (2017) 147 [arXiv: 1703.02981] [INSPIRE].

[35] S. Alexandrov, S. Banerjee and P. Longhi, Rigid limit for hypermultiplets and five-dimensional gauge theories, JHEP 01 (2018) 156 [arXiv:1710.10665] [INSPIRE].

[36] G. Ferlito, A. Hanany, N. Mekareeya and G. Zafrir, 3d Coulomb branch and $5 d$ Higgs branch at infinite coupling, JHEP 07 (2018) 061 [arXiv:1712.06604] [INSPIRE].

[37] P. Jefferson, H.-C. Kim, C. Vafa and G. Zafrir, Towards Classification of 5d SCFTs: Single Gauge Node, arXiv:1705.05836 [INSPIRE].

[38] P. Jefferson, S. Katz, H.-C. Kim and C. Vafa, On Geometric Classification of $5 d$ SCFTs, JHEP 04 (2018) 103 [arXiv:1801.04036] [INSPIRE].

[39] F. Apruzzi, L. Lin and C. Mayrhofer, Phases of $5 d$ SCFTs from M-/F-theory on Non-Flat Fibrations, JHEP 05 (2019) 187 [arXiv:1811.12400] [INSPIRE].

[40] L. Bhardwaj and P. Jefferson, Classifying 5d SCFTs via 6d SCFTs: Rank one, JHEP 07 (2019) 178 [Addendum ibid. 01 (2020) 153] [arXiv:1809.01650] [INSPIRE].

[41] C. Closset, M. Del Zotto and V. Saxena, Five-dimensional SCFTs and gauge theory phases: an M-theory/type IIA perspective, SciPost Phys. 6 (2019) 052 [arXiv:1812.10451] [INSPIRE].

[42] M. Del Zotto and G. Lockhart, Universal Features of BPS Strings in Six-dimensional SCFTs, JHEP 08 (2018) 173 [arXiv: 1804.09694] [INSPIRE].

[43] L. Bhardwaj and P. Jefferson, Classifying 5d SCFTs via 6d SCFTs: Arbitrary rank, JHEP 10 (2019) 282 [arXiv: 1811.10616] [InSPIRE].

[44] L. Bhardwaj, Revisiting the classifications of 6d SCFTs and LSTs, JHEP 03 (2020) 171 [arXiv: 1903.10503] [INSPIRE].

[45] L. Bhardwaj, Dualities of 5d gauge theories from S-duality, JHEP 07 (2020) 012 [arXiv: 1909.05250] [INSPIRE].

[46] L. Bhardwaj, P. Jefferson, H.-C. Kim, H.-C. Tarazi and C. Vafa, Twisted Circle Compactifications of $6 d$ SCFTs, arXiv:1909.11666 [INSPIRE].

[47] L. Bhardwaj, Do all 5d SCFTs descend from 6d SCFTs?, arXiv:1912.00025 [INSPIRE].

[48] L. Bhardwaj and G. Zafrir, Classification of $5 d N=1$ gauge theories, JHEP 12 (2020) 099 [arXiv: 2003. 04333] [INSPIRE].

[49] L. Bhardwaj, More 5d KK theories, arXiv:2005.01722 [InSPIRE].

[50] F. Apruzzi, S. Schäfer-Nameki and Y.-N. Wang, 5d SCFTs from Decoupling and Gluing, JHEP 08 (2020) 153 [arXiv: 1912.04264] [INSPIRE].

[51] F. Apruzzi, C. Lawrie, L. Lin, S. Schäfer-Nameki and Y.-N. Wang, Fibers add Flavor, Part II: 5d SCFTs, Gauge Theories, and Dualities, JHEP 03 (2020) 052 [arXiv:1909.09128] [INSPIRE].

[52] F. Apruzzi, C. Lawrie, L. Lin, S. Schäfer-Nameki and Y.-N. Wang, 5d Superconformal Field Theories and Graphs, Phys. Lett. B 800 (2020) 135077 [arXiv: 1906.11820] [INSPIRE].

[53] F. Apruzzi, C. Lawrie, L. Lin, S. Schäfer-Nameki and Y.-N. Wang, Fibers add Flavor, Part I: Classification of 5d SCFTs, Flavor Symmetries and BPS States, JHEP 11 (2019) 068 [arXiv: 1907.05404] [INSPIRE]. 
[54] C. Closset and M. Del Zotto, On 5d SCFTs and their BPS quivers. Part I: B-branes and brane tilings, arXiv:1912.13502 [INSPIRE].

[55] H.-C. Kim, S.-S. Kim and K. Lee, Higgsing and twisting of $6 d D_{N}$ gauge theories, JHEP 10 (2020) 014 [arXiv: 1908.04704] [INSPIRE].

[56] P. Benetti Genolini, M. Honda, H.-C. Kim, D. Tong and C. Vafa, Evidence for a Non-Supersymmetric 5d CFT from Deformations of $5 d \mathrm{SU}(2)$ SYM, JHEP 05 (2020) 058 [arXiv: 2001.00023] [INSPIRE].

[57] H. Hayashi, P. Jefferson, H.-C. Kim, K. Ohmori and C. Vafa, SCFTs, Holography, and Topological Strings, arXiv:1905.00116 [INSPIRE].

[58] A. Bourget, J.F. Grimminger, A. Hanany, M. Sperling and Z. Zhong, Magnetic Quivers from Brane Webs with O5 Planes, JHEP 07 (2020) 204 [arXiv:2004.04082] [InSPIRE].

[59] A. Bourget, S. Cabrera, J.F. Grimminger, A. Hanany and Z. Zhong, Brane Webs and Magnetic Quivers for SQCD, JHEP 03 (2020) 176 [arXiv:1909.00667] [INSPIRE].

[60] S. Cabrera, A. Hanany and M. Sperling, Magnetic quivers, Higgs branches, and $6 d$ $N=(1,0)$ theories, JHEP 06 (2019) 071 [Erratum ibid. 07 (2019) 137] [arXiv: 1904.12293] [INSPIRE].

[61] S. Cabrera, A. Hanany and F. Yagi, Tropical Geometry and Five Dimensional Higgs Branches at Infinite Coupling, JHEP 01 (2019) 068 [arXiv:1810.01379] [INSPIRE].

[62] H. Hayashi, S.-S. Kim, K. Lee and F. Yagi, Complete prepotential for $5 d \mathcal{N}=1$ superconformal field theories, JHEP 02 (2020) 074 [arXiv: 1912.10301] [INSPIRE].

[63] H. Hayashi, S.-S. Kim, K. Lee and F. Yagi, Rank-3 antisymmetric matter on 5-brane webs, JHEP 05 (2019) 133 [arXiv: 1902.04754] [INSPIRE].

[64] H. Hayashi, S.-S. Kim, K. Lee and F. Yagi, Dualities and 5-brane webs for $5 d$ rank 2 SCFTs, JHEP 12 (2018) 016 [arXiv:1806.10569] [INSPIRE].

[65] H. Hayashi and K. Ohmori, 5d/6d DE instantons from trivalent gluing of web diagrams, JHEP 06 (2017) 078 [arXiv: 1702.07263] [INSPIRE].

[66] H. Hayashi, S.-S. Kim, K. Lee and F. Yagi, 6d SCFTs, 5d Dualities and Tao Web Diagrams, JHEP 05 (2019) 203 [arXiv: 1509.03300] [INSPIRE].

[67] V. Saxena, Rank-two 5d SCFTs from M-theory at isolated toric singularities: a systematic study, JHEP 04 (2020) 198 [arXiv:1911.09574] [INSPIRE].

[68] I. Garozzo, N. Mekareeya, M. Sacchi and G. Zafrir, Symmetry enhancement and duality walls in 5d gauge theories, JHEP 06 (2020) 159 [arXiv:2003.07373] [INSPIRE].

[69] Y. Tachikawa, On the $6 d$ origin of discrete additional data of $4 d$ gauge theories, JHEP 05 (2014) 020 [arXiv: 1309.0697] [INSPIRE].

[70] D.R. Morrison, S. Schäfer-Nameki and B. Willett, Higher-Form Symmetries in 5d, JHEP 09 (2020) 024 [arXiv:2005.12296] [INSPIRE].

[71] M. Dierigl, P.-K. Oehlmann and F. Ruehle, Non-Simply-Connected Symmetries in $6 D$ SCFTs, JHEP 10 (2020) 173 [arXiv: 2005.12929] [INSPIRE].

[72] J. de Boer, R. Dijkgraaf, K. Hori, A. Keurentjes, J. Morgan, D.R. Morrison et al., Triples, fluxes, and strings, Adv. Theor. Math. Phys. 4 (2002) 995 [hep-th/0103170] [INSPIRE].

[73] J.A. Minahan and M. Zabzine, Gauge theories with 16 supersymmetries on spheres, JHEP 03 (2015) 155 [arXiv: 1502.07154] [INSPIRE]. 
[74] K. Polydorou, A. Rocén and M. Zabzine, $7 D$ supersymmetric Yang-Mills on curved manifolds, JHEP 12 (2017) 152 [arXiv:1710.09653] [INSPIRE].

[75] N. Iakovidis, J. Qiu, A. Rocén and M. Zabzine, $7 D$ supersymmetric Yang-Mills on hypertoric 3-Sasakian manifolds, JHEP 06 (2020) 026 [arXiv:2003.12461] [INSPIRE].

[76] D. Gaiotto, G.W. Moore and A. Neitzke, Framed BPS States, Adv. Theor. Math. Phys. 17 (2013) 241 [arXiv: 1006.0146] [InSPIRE].

[77] O. Aharony, N. Seiberg and Y. Tachikawa, Reading between the lines of four-dimensional gauge theories, JHEP 08 (2013) 115 [arXiv: 1305.0318] [INSPIRE].

[78] O. Aharony and E. Witten, Anti-de Sitter space and the center of the gauge group, JHEP 11 (1998) 018 [hep-th/9807205] [INSPIRE].

[79] E. Witten, AdS/CFT correspondence and topological field theory, JHEP 12 (1998) 012 [hep-th/9812012] [INSPIRE].

[80] E. Witten, On flux quantization in M-theory and the effective action, J. Geom. Phys. 22 (1997) 1 [hep-th/9609122] [INSPIRE].

[81] H. Sati, Framed M-branes, corners, and topological invariants, J. Math. Phys. 59 (2018) 062304 [arXiv: 1310.1060] [INSPIRE].

[82] K. Hikami, Decomposition of Witten-Reshetikhin-Turaev invariant: Linking pairing and modular forms, AMS/IP Stud. Adv. Math. 50 (2011) 131 [INSPIRE].

[83] D. Mumford, Tata Lectures on Theta, 1, Modern Birkäuser Classics, Springer, Dordrecht (2007).

[84] I. García-Etxebarria and M. Montero, Dai-Freed anomalies in particle physics, JHEP 08 (2019) 003 [arXiv: 1808.00009] [INSPIRE].

[85] E. Witten, Topological Tools in Ten-dimensional Physics, Int. J. Mod. Phys. A 1 (1986) 39 [INSPIRE].

[86] A. Hatcher, Algebraic Topology, Algebraic Topology, Cambridge University Press (2002).

[87] E. Sharpe, Undoing decomposition, Int. J. Mod. Phys. A 34 (2020) 1950233 [arXiv: 1911.05080] [INSPIRE].

[88] Y. Tanizaki and M. Ünsal, Modified instanton sum in QCD and higher-groups, JHEP 03 (2020) 123 [arXiv: 1912.01033] [INSPIRE].

[89] W. Gu, E. Sharpe and H. Zou, Notes on two-dimensional pure supersymmetric gauge theories, arXiv:2005.10845 [INSPIRE].

[90] G. Barthel and K.H. Fieseler, Invariant divisors and homology of compact complex toric varieties, J. Math. Sci. 82 (1996) 3615.

[91] D. Cox, J. Little and H. Schenck, Toric Varieties, Graduate studies in mathematics, American Mathematical Society (2011).

[92] V. Danilov, The geometry of toric varieties, Russ. Math. Surv. 33 (1978) 97.

[93] S. Friedl, Algebraic Topology I - V.5, https://www.uni-regensburg.de/Fakultaeten/nat_ Fak_I/friedl/papers/2020_friedl-algebraic-topology.pdf (2020).

[94] D.A. Cox, J.B. Little and H.K. Schenck, Toric Varieties, Graduate Studies in Mathematics, AMS (2011). 
[95] S. Reffert, The Geometer's Toolkit to String Compactifications, in Conference on String and M-theory Approaches to Particle Physics and Cosmology, (2007) [arXiv:0706.1310] [INSPIRE].

[96] F. Denef, Les Houches Lectures on Constructing String Vacua, Les Houches 87 (2008) 483 [arXiv:0803.1194] [INSPIRE].

[97] K. Hori, S. Katz, A. Klemm, R. Pandharipande, R. Thomas, C. Vafa et al., Mirror symmetry, vol. 1 of Clay mathematics monographs, AMS, Providence, U.S.A. (2003).

[98] Y.-H. He, R.-K. Seong and S.-T. Yau, Calabi-Yau Volumes and Reflexive Polytopes, Commun. Math. Phys. 361 (2018) 155 [arXiv: 1704.03462] [INSPIRE].

[99] W. Stein et al., Sage Mathematics Software (Version 9.1), The Sage Development Team (2020).

[100] I. García-Etxebarria and B. Heidenreich, S-duality in $\mathcal{N}=1$ orientifold SCFTs, Fortsch. Phys. 65 (2017) 1700013 [arXiv: 1612.00853] [INSPIRE].

[101] J.P. Gauntlett, D. Martelli, J. Sparks and D. Waldram, Sasaki-Einstein metrics on $S^{2} \times S^{3}$, Adv. Theor. Math. Phys. 8 (2004) 711 [hep-th/0403002] [INSPIRE].

[102] O. Aharony, A. Hanany and B. Kol, Webs of $(p, q)$ five-branes, five-dimensional field theories and grid diagrams, JHEP 01 (1998) 002 [hep-th/9710116] [INSPIRE].

[103] P.C. Argyres and M.R. Douglas, New phenomena in SU(3) supersymmetric gauge theory, Nucl. Phys. B 448 (1995) 93 [hep-th/9505062] [inSPIRE].

[104] F. Benini, S. Benvenuti and Y. Tachikawa, Webs of five-branes and $N=2$ superconformal field theories, JHEP 09 (2009) 052 [arXiv:0906. 0359] [INSPIRE].

[105] J. Eckhard, S. Schäfer-Nameki and Y.-N. Wang, Trifectas for $T_{N}$ in 5d, JHEP 07 (2020) 199 [arXiv: 2004.15007] [INSPIRE].

[106] L. Foscolo and M. Haskins, New $g_{2}$-holonomy cones and exotic nearly Kähler structures on $S^{6}$ and $S^{3} \times S^{3}$, Annals Math. 185 (2017) 59 [arXiv:1501.07838] [INSPIRE].

[107] B.S. Acharya and S. Gukov, $M$ theory and singularities of exceptional holonomy manifolds, Phys. Rept. 392 (2004) 121 [hep-th/0409191] [INSPIRE].

[108] G. 't Hooft, On the Phase Transition Towards Permanent Quark Confinement, Nucl. Phys. $B 138$ (1978) 1 [INSPIRE].

[109] R. Bryand and S. Salamon, On the construction of some complete metrices with expectional holonomy, Duke Math. J. 58 (1989) 829 [INSPIRE].

[110] G.W. Gibbons, D.N. Page and C.N. Pope, Einstein Metrics on $S^{3}, R^{3}$ and $R^{4}$ Bundles, Commun. Math. Phys. 127 (1990) 529 [INSPIRE].

[111] M. Atiyah, J.M. Maldacena and C. Vafa, An M-theory flop as a large $N$ duality, J. Math. Phys. 42 (2001) 3209 [hep-th/0011256] [INSPIRE].

[112] B.S. Acharya, Confining strings from $G_{2}$ holonomy space-times, hep-th/0101206 [INSPIRE].

[113] B.S. Acharya and C. Vafa, On domain walls of $N=1$ supersymmetric Yang-Mills in four-dimensions, hep-th/0103011 [INSPIRE]. 